www.nature.com/pj

\title{
The slow relaxation mode: from solutions to gel networks
}

\begin{abstract}
Junfang $\mathrm{Li}^{1}$, To $\mathrm{Ngai}^{2}$ and Chi $\mathrm{Wu}^{2,3}$
In the past, many laser light-scattering experimental results revealed that besides the fast relaxation mode, there existed an additional slow mode in semidilute solutions. This slow mode has been assigned to a variety of origins, but there has been no clear and well-accepted explanation. As the polymer concentration increases, the slow relaxation mode persists in the concentrated region, in melts and in gels in which polymer chains are crosslinked instead of entangled. The slow relaxation mode has also been reported for charged macromolecules in aqueous and nonaqueous solutions. However, it is generally thought to be different in nature from that observed in semidilute neutral polymer solution. In recent years, armed with novel solution preparation methods and some specially designed polymers, we have reexamined the dynamics of polymer chains, especially the slow mode, in semidilute neutral polymer solutions, dilute polyelectrolyte solutions and gels, which are reviewed here. Our results suggest that the slow mode can be qualitatively considered as hindered motions of interacting chains even though the nature of interaction can be very different; namely, from the weak segment-segment interaction in a less good solvent to strong electrostatic interaction among polyelectrolyte chains, and even to chemical crosslinking inside gel networks.
\end{abstract}

Polymer Journal (2010) 42, 609-625; doi:10.1038/pj.2010.59

Keywords: anionic polymerization; laser light scattering; polyelectrolyte; polymer interaction; semidilute; slow relaxation mode; sol-gel transition

\section{INTRODUCTION}

The dynamics of chains in solutions and gels is an old subject of polymer science. Although the behaviors of individual isolated polymer chains in dilute solution under the $\theta$ - and good solvent condition are fairly understood, ${ }^{1-27}$ the slow dynamics of polymer chains in semidilute, concentrated solutions as well as in gel networks still remains a challenging problem since the 1980s. Because of the existence of overlapping and entanglement of polymer chains, new dynamics processes such as interchains interactions and disentanglement come into the problem. Many theoretical and experimental works have been carried out to clarify the properties of these systems. ${ }^{28-75}$ Among these, dynamics and relaxation processes investigated by dynamic laser light scattering (LLS) are of particular interest. In the past, many dynamic LLS experimental results revealed that besides the fast relaxation mode, there existed an additional slow relaxation mode in semidilute solution. It is a widely held view that the fast mode is related to the cooperative diffusion of chain segments between two neighboring entanglement points ('blob'), whereas the slow mode has been assigned to a variety of origins, such as reptation of a clustering of polymer chains through the entangled coils, the scattering vector $(q)$-independent relaxation of a transient network, the $q^{2}$-dependent translational diffusion of large aggregates or even dust particles. Therefore, a clear understanding with regard to the origins of the slow relaxation mode is still lacking. Note that as the polymer concentration increases, the slow relaxation generally persists in concentrated solutions, melts as well as gels in which polymer chains are crosslinked instead of entangled.

The slow relaxation mode has also been commonly reported for nearly all charged macromolecules, including both synthetic and biological polyelectrolytes in aqueous and nonaqueous solutions. The interpretation of the slow mode, especially for those very slow relaxation modes observed in salt-free or low-salt polyelectrolyte solutions, is very controversial. ${ }^{76-95}$ It has been attributed to large multichain domains formed because of electrostatic interaction or some insoluble clusters or even a trace amount of dust particles introduced during the imperfect preparation of polymer solution. Actually, not everyone accepts or recognized such a slow mode, even though it has been repeatedly observed in many dynamic LLS experiments for more than three decades, because of some problems or questions related to previous light-scattering experiments, such as some earlier premature data analysis methods and the preparation of dust-free viscous solutions. As a general phenomenon, which still

\footnotetext{
${ }^{1}$ State Key Laboratory of Organometallic Chemistry, Shanghai Institute of Organic Chemistry, Chinese Academy of Sciences, Shanghai, PR China; ${ }^{2}$ Department of Chemistry, The Chinese University of Hong Kong, Hong Kong, PR China and ${ }^{3}$ Hefei National Laboratory for Physical Sciences at Microsacle, Department of Chemical Physics, University of Science and Technology of China, Anhui, PR China

Correspondence: Dr J Li, State Key Laboratory of Organometallic Chemistry, Shanghai Institute of Organic Chemistry, Chinese Academy of Sciences, Lingling Lu, Shanghai, PR China. E-mail: junfangli@mail.sioc.ac.cn or Professor T Ngai, Department of Chemistry, The Chinese University of Hong Kong, Shatin. N.T., Hong Kong, PR China.

E-mail: tongai@cuhk.edu.hk
}

Received 28 April 2010; revised and accepted 4 June 2010 
remains an important controversial problem in polymer science, it certainly deserves a careful reexamination so that we can find its physical nature. Moreover, advancements of LLS instrumentation, including the full digital time correlator and computer, have made the dynamic study of slow relaxation mode much easier and reliable.

In recent years, by designing, synthesizing and using some novel polymers or sample preparation methods, we have reexamined the dynamics of slow relaxation mode in polyelectrolyte, semidilute solutions and gelling systems by a combination of static and dynamic LLS. ${ }^{75,96-107}$ In this article, we mainly review what has been carried out in our laboratory during the past 10 years. We will outline the details of each system in the following sections by starting with a brief introduction of LLS.

\section{LLS}

In a broad definition, LLS could be grouped as inelastic (for example, Raman, fluorescence and phosphorescence) and elastic (no adsorption) light scattering. However, in polymer and colloid science, light scattering is normally referred to in terms of static (elastic) or dynamic (quasi-elastic) measurements, or both. ${ }^{108}$ Static LLS as a classic and absolute analytical method measures the time-average intensity and it has been long and widely used to characterize both synthetic and natural macromolecules. ${ }^{109}$ On the other hand, dynamic LLS measures the intensity fluctuation. This is where the word dynamic comes from. The visibility of the scattering objects (macromolecules or colloidal particles) in LLS depends on the refractive index difference between the scattering object and the background $\left(\mathrm{d} n=n-n_{0}\right)$.

In the last two decades, owing to the advance of stable laser, ultrafast electronics and personal computers, LLS, especially dynamic LLS, has evolved from a very special instrument for physicists and physical chemists to a routine analytical tool in polymer laboratories or even to a daily quality-control device in production lines. Commercially available research-grade LLS instruments nowadays are normally capable of making static and dynamic measurements simultaneously for studies of colloidal particles in suspensions or macromolecules in solutions as well as in gels and viscous media. In our laboratory, we have used a slightly modified commercial LLS spectrometer (ALV/DLS/ SLS-5022f, ALV GmbH, Langen, Germany) equipped with a cylindrical 22-mW UNIPHASE He-Ne laser $\left(\lambda_{0}=632 \mathrm{~nm}\right.$, JDS Uniphase Corp., Milpitas, CA, USA) as the light source and a sensitive Avalanche Photo Diode (APD) detector. The incident light beam was vertically polarized with respect to the scattering plane and the intensity was regulated with a beam attenuator (Newport Corp., Mountain View, CA, USA, M-925B) so as to avoid localized heating in the light-scattering cuvette. It is worth mentioning that many reviews, books, proceedings and chapters have been published on the details of LLS instrumentation and theory. Herein, we will concentrate only on the relevant details.

When a light beam is incident on a solution, and the solvent refractive index is different from that of the solute (macromolecules or colloidal particles), the incident light is scattered by each illuminated macromolecule or colloidal particle in all directions. ${ }^{9-11}$ If all scattering species are stationary, the scattered light intensity at each direction would be a constant, that is, independent of time. However, in reality, all the solute in solution are undergoing constant Brownian motions, which leads to both fluctuations of the scattered intensity pattern on the detection plane and the fluctuations of net scattered light intensity, $I(t)$, with time in sufficiently small detector area. The fluctuation rates can be related to different relaxation processes such as translational and rotational diffusions as well as internal motions of the macromolecules. The faster the relaxation process, the faster the intensity fluctuations will be. In the homodyne or self-beating system, the intensity fluctuations can relate to the dynamic pair correlating of the scattering species:

$$
\left\langle\delta I_{q}(0) \delta I_{q}(t)\right\rangle=|S(q, t)|^{2}=\langle S(q)\rangle^{2}\left[1+\beta_{\text {app }}\left|g_{\text {solute }}^{(1)}(q, t)\right|^{2}\right]
$$

where $q=|\vec{q}|=\frac{4 \pi n}{\lambda_{0}} \sin \left(\frac{\theta}{2}\right)$ with $n, \lambda_{0}$ and $\theta$ are the solvent refractive index, the wavelength of the light in vacuum and scattering angle, respectively, $\beta$ is the coherent factor depending on the detection optics, $S(q, t)$ is the dynamic scattering factor, $S(q)$ is the structure factor, $g^{(1)}(q, t)$ is the normalized field-field time correlation functions, defined as:

$$
\begin{aligned}
& \left\langle E(0, q) E^{*}(t, q)\right\rangle /\left\langle E(0) E^{*}(0)\right\rangle, \text { If } t \rightarrow \infty, \quad g^{(1)}(q, t)=0, \text { and } \\
& S(q, t=\infty)=S(q)=\int \exp \left[\mathrm{i} \vec{q} \cdot\left(\vec{r}_{\mathrm{mi}}-\vec{r}_{\mathrm{nj}}\right)\right] \mathrm{d} N \mathrm{~d} \vec{r}
\end{aligned}
$$

For polymer in dilute solution without long-range pair interaction, the structure factor can relate to molar mass and form factor of the scattering particle by the follow equation:

$$
S(q) \propto N_{\mathrm{p}} N P(q) \propto C M\left(1-2 C M A_{2}+\ldots\right)\left(1-\frac{q^{2}}{3} R_{\mathrm{g}}^{2}+\ldots\right)
$$

where $N_{\mathrm{p}}, N, C, M, A_{2}$ and $R_{\mathrm{g}}$ are the number of polymer chain, the number of monomer in each polymer, the concentration, molar mass of polymer, the second virial coefficient and the radius of gyration, respectively. In static LLS, the excess absolute time-averaged scattered light intensity, known as the excess Rayleigh ratio $R_{v v}(q)$, of a dilute polymer solution at concentration $C\left(\mathrm{~g} \mathrm{ml}^{-1}\right)$ is related to the weight average molar mass $M_{\mathrm{w}}$, the root-mean square $z$-average radius of gyration $\left\langle R_{\mathrm{g}}{ }^{2}\right\rangle_{z}^{1 / 2}$ (or written as $\left\langle R_{\mathrm{g}}\right\rangle$ ), and the scattering vector $q$ as:

$$
\frac{K C}{R_{v v}(q)} \approx \frac{1}{M_{\mathrm{w}}}\left(1+\frac{1}{3}\left\langle R_{\mathrm{g}}^{2}\right\rangle q^{2}-\ldots\right)+2 A_{2} C-\ldots
$$

where $K=4 \pi^{2} n^{2}(\mathrm{~d} n / \mathrm{d} C)^{2} /\left(N_{\mathrm{A}} \lambda_{0}^{4}\right)$ with $N_{\mathrm{A}}$ and $\mathrm{d} n / \mathrm{d} C$ being the Avogadro number and the specific refractive index increment, respectively, This is the most basic equation in static LLS. With $R_{v v}(q)$ measured over a series of $C$ and $q$, one can obtain $\left\langle R_{\mathrm{g}}\right\rangle$ and $A_{2}$, respectively, from the slopes of $\left[K C / R_{v v}(q)\right]_{C \rightarrow 0}$ vs $q^{2}$ and $[K C /$ $\left.R_{v v}(q)\right]_{q \rightarrow 0}$ vs $C$; and $M_{\mathrm{W}}$ from $\left[K c / R_{v v}(q)\right]_{C \rightarrow 0, q \rightarrow 0}$. The Zimm plot, that is, $K C / R_{v v}(q)$ vs $\left(q^{2}+\mathrm{k} C\right)$ with $\mathrm{k}$ being an adjustable constant, allows both $q$ and $C$ extrapolations to be made on a single grid.

In dynamic LLS, the intensity-intensity time auto-correlation function $G^{(2)}(q, t)$, defined as $\langle I(q, 0) I(q, t)\rangle /\langle I(q)\rangle^{2}$, in the homodyne mode or self-beating mode was measured, where $t$ is the delay time and $\langle I(q)\rangle$ is the time-average scattering intensity, that is, the measured baseline. $G^{(2)}(q, t)$ is related to $g^{(1)}(t, q)$ by the Siegert relation as:

$$
G^{(2)}(q, t)=A\left[1+\beta\left|g^{(1)}(q, t)\right|^{2}\right]
$$

where $A$ is the measured baseline. For monodispersed spherical scatters, $\left|g^{(1)}(q, t)\right|$ is theoretically represented as an exponential decay function:

$$
\left|g^{(1)}(q, t)\right|=G \exp (-\Gamma t)
$$

where $G$ and $\Gamma$ are the factor of proportionality and the line width, respectively, and $\Gamma=1 / \tau$, the characteristic decay time representing the rate of dynamic relaxation. For a polydispersed dynamic relaxation, equation (6) may be generalized as:

$$
\left|g^{(1)}(q, t)\right|=\int_{0}^{\infty} G(\Gamma) \mathrm{e}^{-t \Gamma} \mathrm{d} \Gamma
$$


where $G(\Gamma)$ is called the line-width distribution and $G(\Gamma) \mathrm{d} \Gamma$ is the statistic weight of the dynamic relaxation, which possess line width or related to the polymer distribution in dilute polymer solution.

The Laplace inversion of each measured $G^{(2)}(q, t)$ can lead to one $G(\tau)$ on the basis of equations 5 and 7. For a diffusive relaxation, $\Gamma(1 / \tau)$ is related to the translational diffusion coefficient $D$ by $\left(\Gamma / q^{2}\right)_{c \rightarrow 0, q \rightarrow 0} \rightarrow D$, so that $G(\Gamma)$ can be converted into a transitional diffusion coefficient distribution $G(D)$ or further a hydrodynamic radius distribution $f\left(R_{\mathrm{h}}\right)$ applying the Stokes-Einstein equation, $R_{\mathrm{h}}=\left(k_{\mathrm{B}} T / 6 \pi \eta\right) / D$, where $k_{\mathrm{B}}, T$ and $\eta$ are the Boltzmann constant, the absolute temperature and the solvent viscosity, respectively. Note that $\eta$ is local viscosity. In general, we cannot conclude that the slower mode should be caused by larger scattering object if the local viscosity is not uniform.

We should note that the Laplace inversion is an ill-conditioned problem because of the bandwidth limitation of photon correlation instruments, some unavoidable noises in the measured time correlation function, and a limited number of data points. Sometimes we used a combination of two stretch exponential functions to fit the correlation function:

$$
\left[G^{(2)}(q, t)-A\right] / A=\beta_{\text {apparent }}\left\{A_{\mathrm{f}} \exp \left[-\left(t / \tau_{\mathrm{f}}\right)^{\delta_{\mathrm{f}}}\right]+A_{\mathrm{s}} \exp \left[-\left(t / \tau_{\mathrm{s}}\right)^{\delta_{\mathrm{s}}}\right]\right\}^{2}
$$

where $\beta_{\text {apparent }} \leqslant \beta$, an apparent coherent factor, $A_{\mathrm{f}}$ and $A_{\mathrm{s}}$ are intensity contributions of the fast and slow modes, respectively, and $A_{\mathrm{f}}+A_{\mathrm{s}}=1$. The details of the data analysis could be referred to the articles. $^{97-107,110,111}$

\section{The slow mode in semidilute neutral polymer solutions}

When the concentration of polymer is higher than the overlap concentration $\left(C^{\star}\right)$, polymer chains start to 'touch' each other and the solution enters the semidilute regime. $C^{*}$ has been differently defined as $3 M /\left(4 \pi N_{\mathrm{A}} R_{\mathrm{g}}{ }^{3}\right)$, or $M /\left(2^{3 / 2} N_{\mathrm{A}} R_{\mathrm{g}}{ }^{3}\right)$, and $[\eta]^{-1}$, where $M, R_{\mathrm{g}}$, $N_{\mathrm{A}}$ and $[\eta]$ are the molar mass, the radius of gyration of polymer chains, the Avogadro constant and the intrinsic viscosity, respectively. The difference of $C^{\star}$ calculated based on these definitions could be as large as five times. ${ }^{112,113}$ On the other hand, at the entanglement concentration $\left(C_{\mathrm{e}}\right)$, polymer chains start to interwind with each other, where $C_{\mathrm{e}}$ can be estimated from the ratio of the chain length $(L)$ and the entanglement length $\left(L_{\mathrm{e}}\right) \cdot{ }^{114} C_{\mathrm{e}}$ is higher than $C^{\star}$. Note that for chains shorter than $L_{\mathrm{e}}$, they are not able entangle with each other even at higher concentrations.

The mean-field theory can effectively describe some properties of polymer solutions over the whole concentration range, but fails to explain other static and dynamic properties because of strong intraand interchain interaction among covalently bonded monomers. Using the concept of a 'blob', the reptation and tube model, de Gennes and colleagues developed some scaling laws to predict various properties of semidilute solutions. ${ }^{114-117}$

In a transient gel model, there exists only one characteristic length or one dynamic process in semidilute solutions if the solvent is thermodynamically good or athermal. In other words, the thermal energy $\left(k_{\mathrm{B}} T\right)$ can only agitate one short segment of the chain so that its gravity center undergoes a random Brownian motion inside a confined volume. The static and dynamic correlation lengths $\left(\xi_{\mathrm{S}}\right.$ and $\left.\xi_{\mathrm{D}}\right)$ can be measured from the angular dependence of the average scattering intensity and the average cooperative diffusion coefficient $\left(D_{\mathrm{C}}\right)$, respectively. Previous studies are well represented by two master curves: $\xi_{\mathrm{S}} \sim C^{-0.72 \pm 0.01}$ and $\xi_{\mathrm{D}} \sim C^{-0.70 \pm 0.01}$, slightly deviated from $\xi \sim C^{-0.75}$ predicted in theories. ${ }^{28,112}$ For a distance longer than $\xi$, the segment-segment interaction is 'completely' screened out in an athermal solvent. ${ }^{116,118,119}$

Besides the fast relaxation mode, an additional slow relaxation mode has also been observed in the intensity-intensity time correlation functions of various semidilute polymer solutions. ${ }^{28-39}$ This slow relaxation mode was assigned to different physical origins, such as the reptation of the entire chain inside a 'tube' made of its surrounding chains, ${ }^{114,115,117}$ the scattering vector $(q)$-independent relaxation of a transient network, ${ }^{29,34,35}$ the $q^{2}$-dependent translational diffusion of large aggregates or even dust particles, ${ }^{32,33,37,38,112}$ and internal motions of large transient chain clusters. ${ }^{92}$ Later, it was realized that the reptation is not observable in dynamic LLS because of its nature for homopolymer system.

Theoretically, Brochard and de Gennes ${ }^{40}$ attributed $D_{\mathrm{C}}$ to the osmotic modulus $\left(M_{\pi}\right)$, the elastic modulus $\left(M_{0}\right)$ and the friction coefficient $(\zeta)$ as $D_{\mathrm{C}}=\left(M_{\pi}+M_{0}\right) / \zeta C$. Adam and Delsanti ${ }^{34,35}$ allowed the transient gel network to relax and assigned the $q$-independent slow mode with a viscoelastic nature and a characteristic time $\left(\tau_{\mathrm{r}}\right)$. On the other hand, Wang et al. ${ }^{41-46}$ related the slow relaxation to the viscoelasticity under the $\Theta$ condition from the osmotic pressure fluctuation without using the concept of a transient gel network. The cooperative diffusion and the viscoelastic effect are generally mixed, depending on the frequency and a coupling parameter $(\beta)$. $\beta=(C / \rho)\left(V_{\mathrm{S}}-V_{\mathrm{P}}\right) / V_{\mathrm{S}}$, proportional to the difference between partial specific volumes of polymer and solvent $\left(V_{S}-V_{\mathrm{P}}\right)$ and $\rho$ is the solution density. The influence of longitudinal modulus on the concentration fluctuation leads to a broad distribution. ${ }^{44-46}$ If $\beta=1$, there exits only one stress-relaxation mode. $D_{\mathrm{C}}$ becomes identical to that predicted in the transient gel network model. ${ }^{40,114,115}$

Experiments around the $\Theta$ condition indicate that the solvent quality has a profound effect on the slow relaxation. ${ }^{48-54}$ Actually, not everyone accepts or recognizes such a slow mode, even though it has been repeatedly observed for more than three decades. In the last two decades, advancements of LLS instrumentation, including the full digital time correlator and computer, have made the dynamic study of semidilute solutions much easier and more reliable, but the preparation of dust-free viscous semidilute solutions still remains an experimental challenge.

Traditionally, viscous dust-free semidilute solutions are prepared by slow evaporation of solvent from a dust-free dilute solution (the increase of polymer concentration). Figure 1 shows how the polymer concentration $(C)$ of four polystyrene (PS) standards increases with the evaporation time $(t)$. The inset shows that the time-average excess scattering intensity $\left(\langle I\rangle_{q \rightarrow 0}=\langle I\rangle_{\text {solution }}-\langle I\rangle_{\text {solvent }}\right)$ decreases as $C$ increases, following a scaling of $\langle I\rangle_{q \rightarrow 0} \propto C^{-0.25 \pm 0.05}$. For a polymer solution, $\langle I\rangle_{q \rightarrow 0} \propto\left(k_{\mathrm{B}} T / V\right) C /(\partial \pi / \partial C)$, where $V$ is the volume and $\partial \pi / \partial C$ is the osmotic compressibility. In dilute solution, $\partial \pi / \partial C=N_{A} k_{\mathrm{B}} T / M$ and then $\langle I\rangle_{q \rightarrow 0} \propto C$. In semidilute solutions, $\partial \pi / \partial C \propto C^{-5 / 4}$ so that $\langle I\rangle_{q \rightarrow 0} \propto C^{-1 / 4}$. The scaling in the inset agrees well with the prediction and shows that solvent evaporation gradually induces the dilute polymer solution to the semidilute regime. As expected, only one fast relaxation mode is observed over the entire concentration range, consistent with some of the previous studies. ${ }^{112,116}$ However, it is worth mentioning that the whole process lasted for about 1 year. Even using such a painful process, one still faces a concentration gradient problem, that is, the upper layer is likely more concentrated than the bottom layer.

On the basis of the definition of $C^{\star}$, one can also change $R_{\mathrm{g}}$ or $M$ to switch a solution from dilute to semidilute for a fixed polymer concentration. One interesting advantage of the in situ change of $R_{\mathrm{g}}$ or $M$ or both inside an LLS cell is that the polymer solution can 


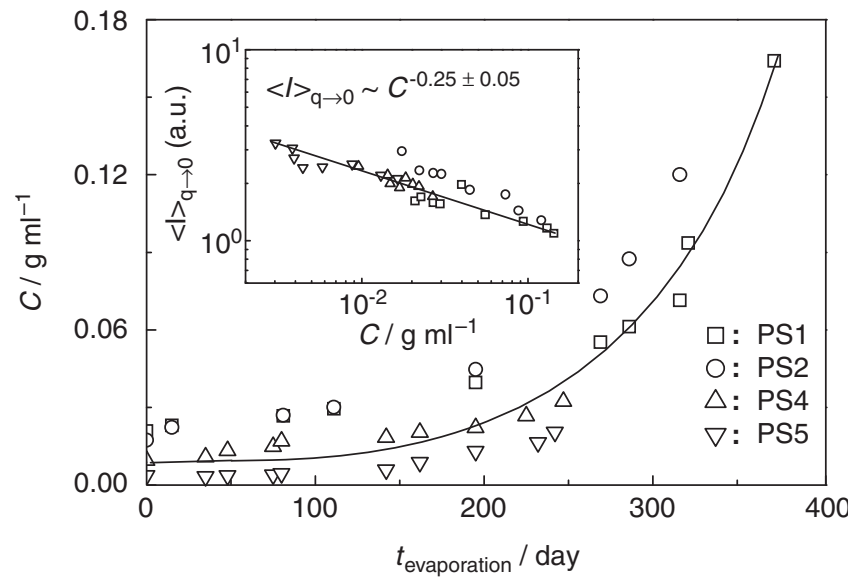

Figure 1 Evaporation time dependence of concentration $(C)$ of four PS standards (PS1, PS2, PS4 and PS5 in Li et al. ${ }^{75}$ ) in toluene. The inset shows concentration dependence of average scattering intensity $\left(\langle I\rangle_{q \rightarrow 0}\right)$ as scattering vector $(q)$ approaches zero. Reproduced with permission from Li et al. ${ }^{75}$ Copyright (2008) American Chemical Society.

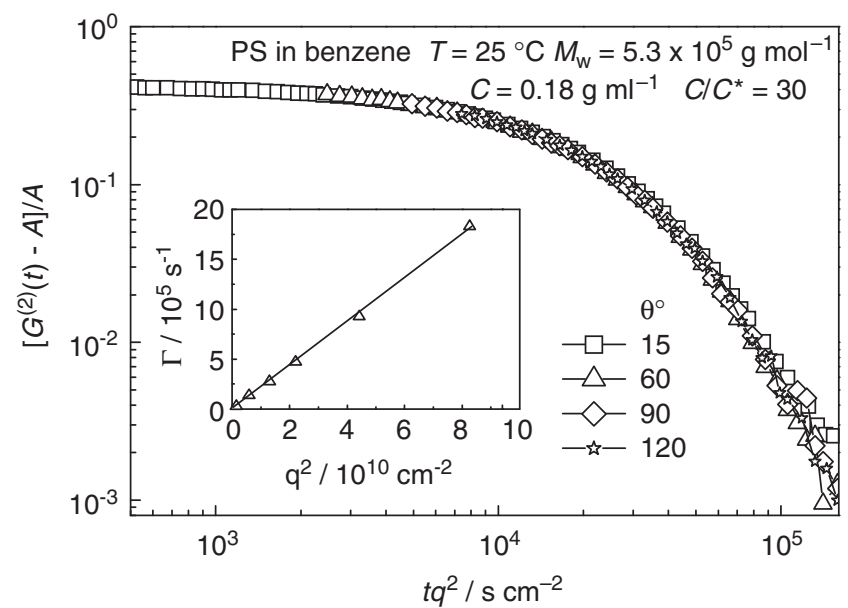

Figure 2 Scattering vector dependence of intensity-intensity time correlation functions of PS in benzene, where delay time $(t)$ is scaled with $q^{2}$. The inset shows scattering vector dependence of average line width. Reproduced with permission from Li et al. ${ }^{75}$ Copyright (2008) American Chemical Society.

completely avoid possible problems of dust, solubility and inhomogeneity. To achieve that, we used anionic living polymerization ${ }^{75}$ to prepare dust-free semidilute/concentrated solutions (up to $30 \%$ ) of narrowly distributed PS chains directly inside the LLS cell with a high vacuum stopcock, starting from a dust-free monomer solution. Subsequently, we comparatively reexamined the solution dynamics of PS chains in both good solvent (benzene) and a less good/ $\theta$ solvent (cyclohexane).

For the solution studied in Figure 2, we terminated living PS chains and measured its weight average molar mass $\left(M_{\mathrm{w}}\right)$ after the end of polymerization. We found that $M_{\mathrm{w}}$ is $\sim 5.3 \times 10^{5} \mathrm{~g} \mathrm{~mol}^{-1}$ and the polymer concentration is $0.18 \mathrm{~g} \mathrm{ml}^{-1}$, that is, $C / C^{\star} \sim 30$. Importantly, Figure 2 shows that even with such a high concentration, there is still only one fast relaxation mode, agreeing well with the prediction in an athermal solvent. The scaling of the delay time $(t)$ with $q^{2}$ makes the measured time correlation functions at different scattering angles collapse into one curve. The inset shows that the average line width

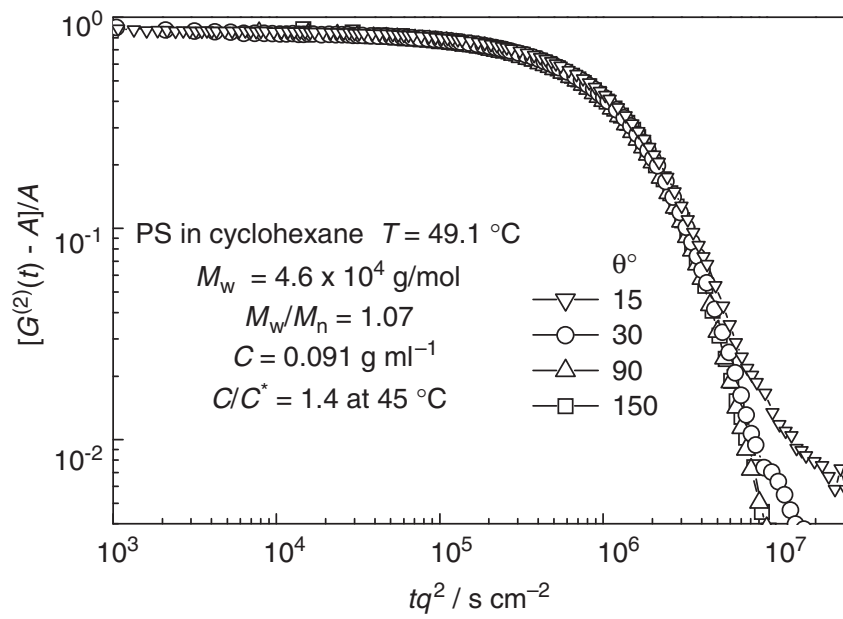

Figure 3 Scattering vector dependence of intensity-intensity time correlation functions of PS in cyclohexane, where delay time $(t)$ is scaled with $q^{2}$. Reproduced with permission from $\mathrm{Li}$ et al. ${ }^{75}$ Copyright (2008) American Chemical Society.

$\left(\langle\Gamma\rangle_{\mathrm{f}}=1 /\langle\tau\rangle_{\mathrm{f}}\right)$ is a linear function of $q^{2}$, passing through the origin, a characteristic of diffusive relaxation. This fast relaxation mode could be modeled as the cooperative motions of the 'blobs' confined inside a 'tube' made of its surrounding chains. Such a fast relaxation mode is also independent of the solution temperature $(T)$ after the scaling of the delay time $(t)$ with $T$ and the solvent viscosity, which is expected because benzene is an athermal solvent for PS in the temperature range studied.

With the same prepared method, we further studied the solution dynamics of PS in a less good $/ \theta$ solvent, cyclohexane. As expected, there is only one fast relaxation mode in cyclohexane when the solution is dilute, independent of the scattering angle. As soon as the solution becomes semidilute, that is, $C / C^{\star}>1$, an additional slow relaxation mode appears in the measured intensity-intensity time correlation function. The analysis of such a time correlation function on the basis of equation (8) leads to two characteristic relaxation times $\left(\langle\tau\rangle_{\mathrm{f}}\right.$ and $\left.\langle\tau\rangle_{\mathrm{s}}\right)$. Figure 3 shows that the slow mode becomes more obvious at smaller scattering angles. Note that in LLS, the observation length is directly proportional to $1 / q$, that is, large objects are more obvious at smaller scattering angles. Also note that here $C / C^{\star}$ is only $\sim 1.4$ at $45^{\circ} \mathrm{C}$ and $C / C_{\mathrm{e}} \sim 0.1$ so that most of the chains are not entangled with each other. In a less good solvent, those entangled chains behave like large transient clusters within the delay time window in dynamic LLS due to relatively strong segment-segment interaction in comparison with in an athermal solvent. Moreover, we found that the characteristic line width $\left(\left\langle\Gamma_{\mathrm{s}}\right\rangle\right)$ is scaled to $q$ as $\left\langle\Gamma_{\mathrm{s}}\right\rangle \sim q^{3}$, indicating that the slow mode reflects some internal motions of large scattering objects. The concentration dependence of the scaling exponent $\left(\alpha_{\mathrm{s}}\right)$ between $\left\langle\Gamma_{\mathrm{s}}\right\rangle$ and $q$ will be discussed more in the follow section.

As the solvent quality also affects the solution dynamics, we studied the temperature dependence of the slow relaxation mode in cyclohexane. Figure 4 shows typical plots of the temperature dependence of intensity-intensity correlation function for the PS in cyclohexane, and Figure 5 shows the corresponding plots of characteristic relaxation time distribution function analyzed by the CONTIN method. It clearly reveals that at a low scattering angle, the decreases of the solution temperature makes the slow mode less obvious and results in a decrease of its intensity contribution (the peak area). At $37^{\circ} \mathrm{C}$, the 


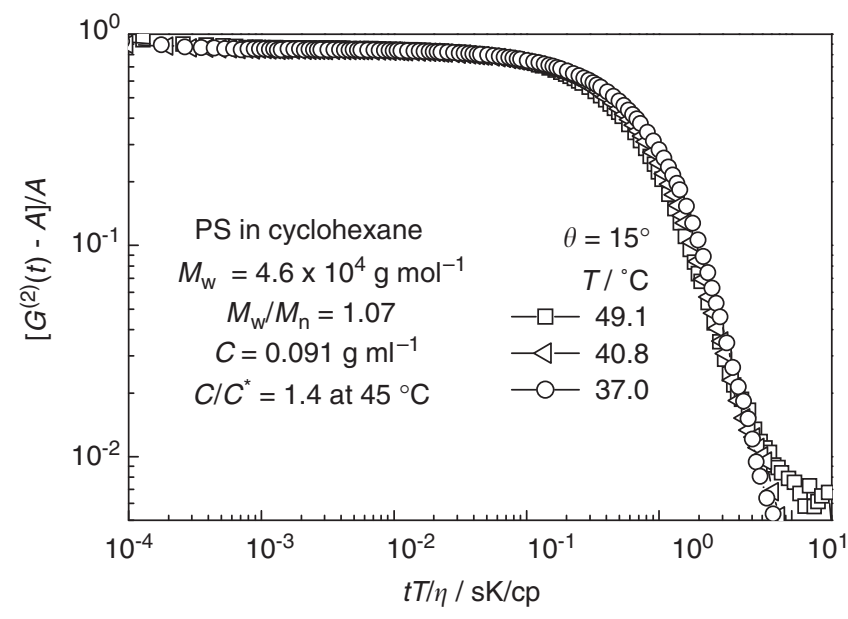

Figure 4 Temperature dependence of intensity-intensity time correlation functions of PS in cyclohexane, where delay time $(t)$ is scaled with solution temperature $(T)$ and solvent viscosity $(\eta)$. Reproduced with permission from Li et al. ${ }^{75}$ Copyright (2008) American Chemical Society.

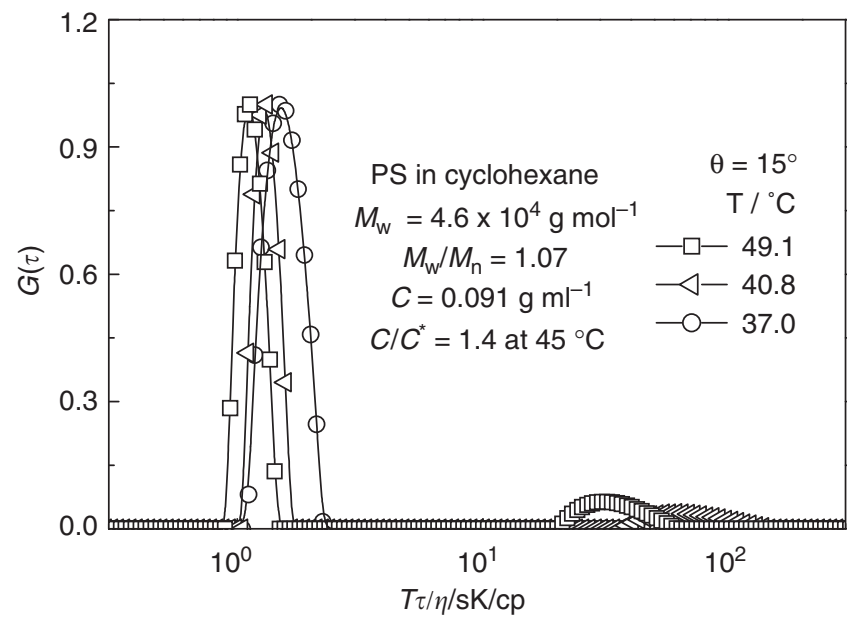

Figure 5 Temperature dependence of characteristic relaxation time distributions $G(\tau)$ of PS in cyclohexane, where characteristic relaxation time $(\tau)$ is scaled with solution temperature $(T)$ and solvent viscosity $(\eta)$. Reproduced with permission from Li et al. ${ }^{75}$ Copyright (2008) American Chemical Society.

slow mode completely disappears in the whole angular range and only one fast mode is observable. As discussed before, the decrease of the solution temperature makes the solvent quality poorer and the segment-segment interaction stronger so that PS chains contract as the temperature decreases, resulting in an increase of $C^{\star}$, which can switch a solution from semidilute to dilute for a given $C$, that is, from $C / C^{\star}>1$ to $C / C^{\star}<1$ when $C / C^{\star}$ is not higher at higher temperature.

Figure 6 shows that for a more concentrated solution, that is, $C / C^{\star}=12$ or more precisely $C / C_{\mathrm{e}}>1$, the fast mode is collapsed together when the decay time $(t)$ is scaled by $q^{2}$, revealing its diffusive nature. In contrast with Figure 3, here the slow mode becomes more obvious at larger angles, which is different from those observed in the above lower concentration region. It implies that the slow mode is not related to some large scattering objects. Further increase of the polymer concentration makes the slow mode even visible at low scattering angles, as shown in Figure 7 . Here, $C / C^{\star}>50$ and the solution appears like a gel without any visible flow within a short time, but the solution is homogeneous and its scattering intensity is

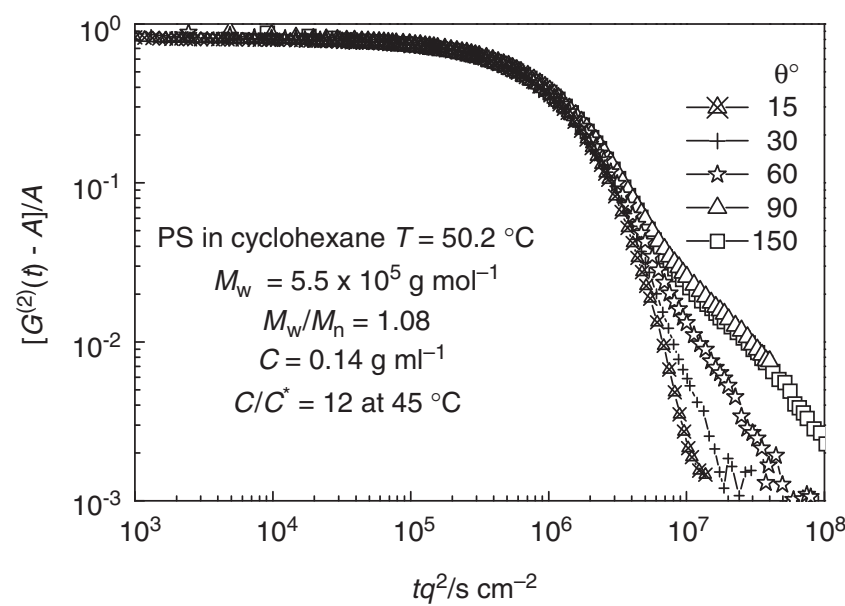

Figure 6 Scattering vector dependence of intensity-intensity time correlation functions of PS in cyclohexane, where delay time $(t)$ is scaled with $q^{2}$. Reproduced with permission from Li et al. ${ }^{75}$ Copyright (2008) American Chemical Society.

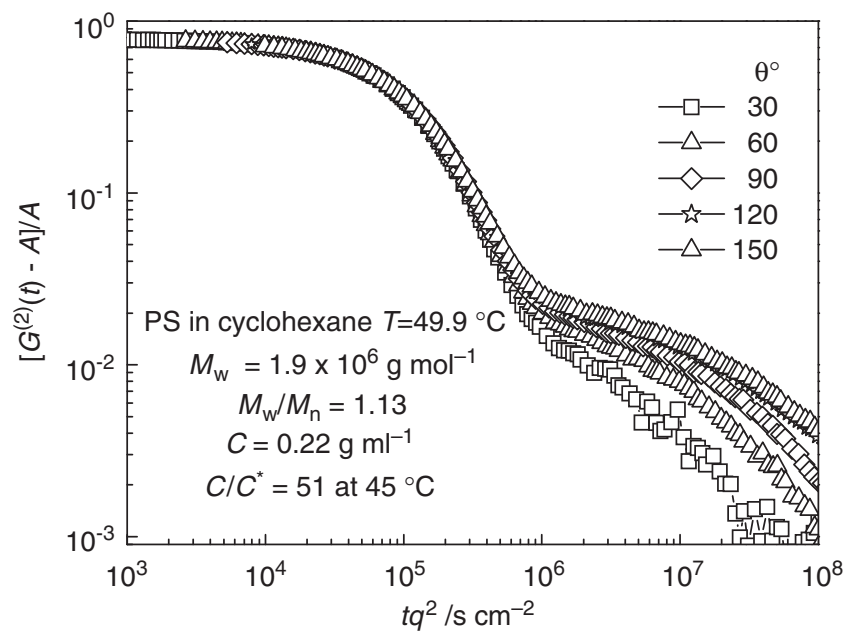

Figure 7 Scattering vector dependence of intensity-intensity time correlation functions of PS in cyclohexane (Solution 10 in Li et al. ${ }^{75}$ ), where delay time $(t)$ is scaled with $q^{2}$. Reproduced with permission from Li et al. ${ }^{75}$ Copyright (2008) American Chemical Society.

independent of the position of the scattering volume inside the solution. Figure 7 , once again shows that the fast mode collapses together after delay time $(t)$ is scaled by $q^{2}$, indicating that it remains diffusive. At the same time, Figure 8 shows that for a given scattering angle, the slow mode become slower and its intensity contribution $\left(A_{\mathrm{s}}\right)$ increases as the solution temperature decreases. Note that the decrease of the solution temperature has two opposite effects on the slow mode. On the one hand, it increases the segment-segment interaction and enhances the slow mode; on the other hand, the chain contraction disentangles the transient clusters. When the latter is dominant, the slow mode becomes weak or even disappears at lower solution temperatures. However, when $C$ is much greater than $C_{\mathrm{e}}$, the chain contraction cannot switch the solution from semidilute to dilute so that decreasing the solution temperature only increases the segment-segment interaction. This presumably is the reason why the slow mode becomes slower and more visible at lower temperatures as shown in Figure 8. 


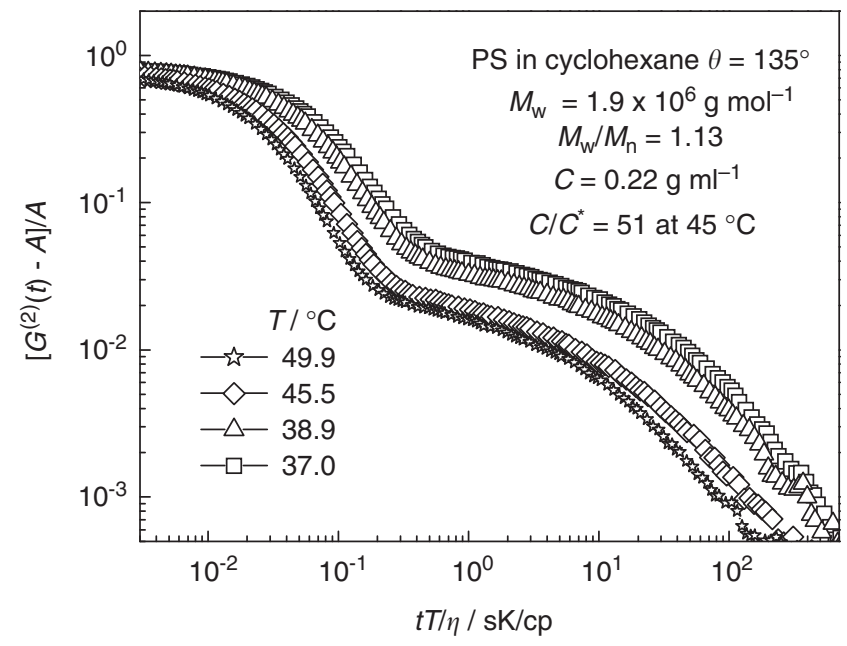

Figure 8 Temperature dependence of intensity-intensity time correlation functions of PS in cyclohexane, where delay time $(t)$ is scaled with solution temperature $(T)$ and solvent viscosity $(\eta)$. Reproduced with permission from Li et al. ${ }^{75}$ Copyright (2008) American Chemical Society.

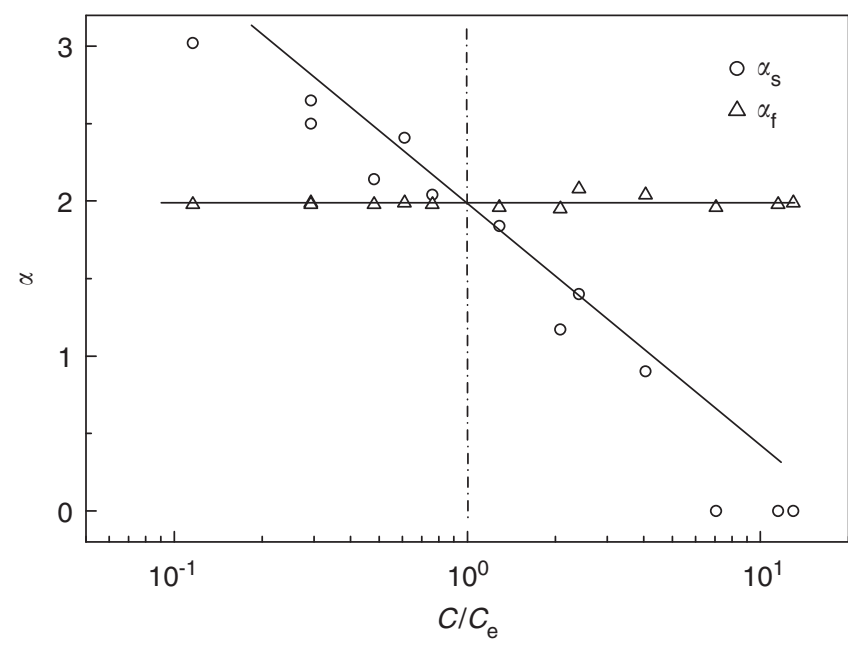

Figure 9 Polymer concentration dependence of two scaling exponents $\left(\alpha_{f}\right.$ and $\alpha_{s}$ ) in $\langle\Gamma\rangle_{f} \propto q^{\alpha f}$ and $\langle\Gamma\rangle_{s} \propto q^{\alpha s}$ for PS in cyclohexane (a good solvent at $45^{\circ} \mathrm{C}$ ). Reproduced with permission from Li et al. ${ }^{75}$ Copyright (2008) American Chemical Society.

Figure 9 summarizes the concentration dependence of the scaling exponents $\left(\alpha_{\mathrm{f}}\right.$ and $\left.\alpha_{\mathrm{s}}\right)$ in $\langle\Gamma\rangle_{\mathrm{f}} \propto q^{\alpha f}$ and $\langle\Gamma\rangle_{\mathrm{s}} \propto q^{\alpha s}$ for PS in cyclohexane solution. As expected, for the fast mode, $\langle\Gamma\rangle_{\mathrm{f}} \propto q^{2}$ over the entire concentration range studied, reflecting the diffusive Brownian motion of the gravity center of each segment (blob). In contrast, for the slow mode, $\alpha_{\mathrm{s}}$ decreases as $C / C_{\mathrm{e}}$ increases. We can roughly divide Figure 9 into two concentration regions (left and right) using $\alpha_{\mathrm{s}}=2$ as a reference (the dashed line). On the left $\left(C / C_{\mathrm{e}} \leqslant 1\right)$, the slow mode is more visible at smaller scattering angles, but in the right region $\left(C / C_{\mathrm{e}}>1\right)$, its intensity contribution to $G(\tau)$ increases with the scattering angle. As mentioned before, the LLS theory tells us that larger objects scatter more light at a lower scattering angle. Therefore, in the left region, the slow mode should be related to scattering objects larger or comparable with $1 / q$; whereas in the right region, it is related to scattering objects smaller than $1 / q$. The LLS theory also tells us that
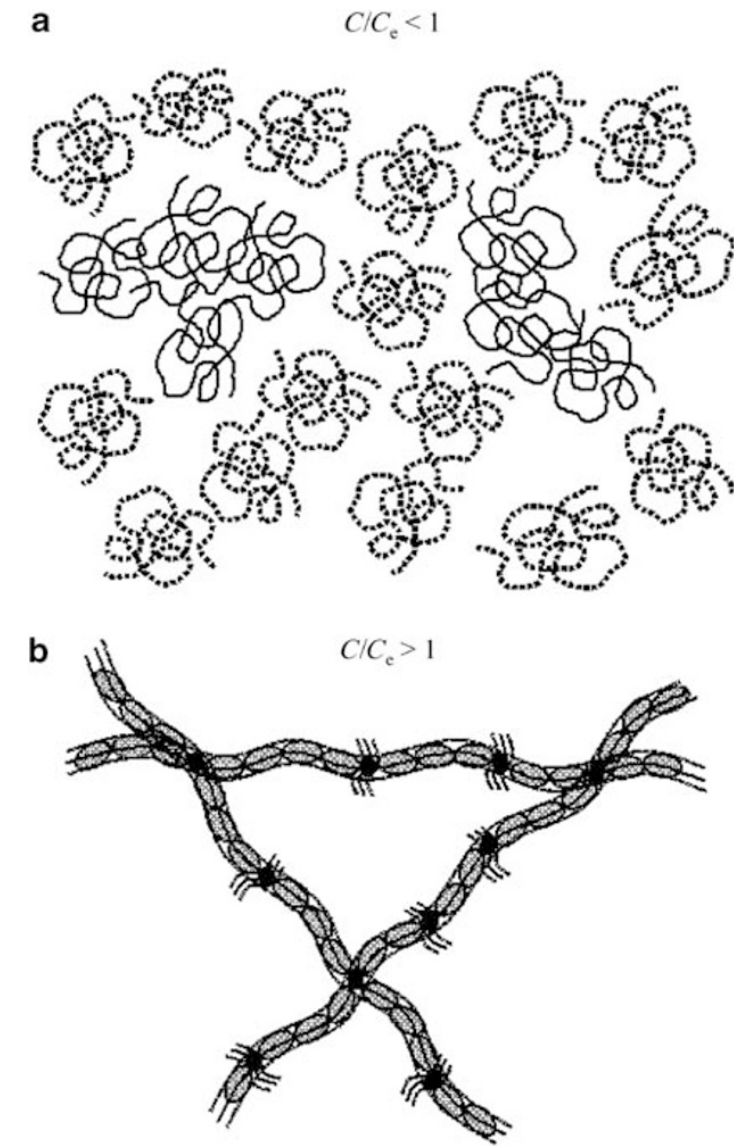

Scheme 1 Semidilute polymer solutions in a less good solvent in different concentration regions. (a) $C / C_{\mathrm{e}}<\sim 1$, where individual chains and 'clusters' have the same microenvironment, but different sizes; and (b) $C / C_{\mathrm{e}}>1$, where all the chains are overlapped and entangled with each other. Light and dark spheres represent 'blobs' between two entanglement points and around entanglement points, respectively. Reproduced with permission from Li et al. ${ }^{75}$ Copyright (2008) American Chemical Society.

when the scaling exponent $(\alpha)$ between the characteristic line width $(\langle\Gamma\rangle)$ and the scattering vector $(q)$ is $>2$, we are observing a mixture of diffusion and internal motions of larger scattering objects. However, it is not clear why $\alpha$ is $<2$ in a three-dimensional space.

In short, the comparative studies of different solvents, concentrations and temperatures convincingly demonstrated that the slow mode is real in semidilute solution. Previously, we speculated that the slow mode might be related to some long-range density fluctuation. The recent results indicate that for $C / C_{\mathrm{e}} \leqslant 1$, the slow mode is related to some large structures (scattering objects or long-range density fluctuation), as it is more visible at lower scattering angles and the scaling exponent $\left(\alpha_{s}\right)$ is higher than 2. Such large structures could be attributed to a few transient clusters made of lightly entangled chains due to relatively stronger segment-segment interaction in a less good solvent. In this case, most of the chains are not entangled with each other and they coexist with those transient 'clusters', as schematically shown in Scheme 1a. Strictly speaking, these solutions are not semidilute but a transitional region between dilute and semidilute solutions. Our results reveal that one should use $C_{\mathrm{e}}$ instead of $C^{\star}$ to mark the real boundary of semidilute solutions. In the transitional region $\left(C^{\star} \leqslant C \leqslant C_{\mathrm{e}}\right)$, it is these transient chain entanglements that make the solution microscopically inhomogeneous over a short time scale $\left(\sim 10^{-3} \mathrm{~s}\right)$. 
When $C>C_{\mathrm{e}}$, all the chains inside the solution entangle with each other to form one huge 'cluster'. Each chain could be visualized to arrest inside a 'tube' made of its surrounding chains within a short time and 'divided' into a number of segments (blobs) with a dimension limited by the tube diameter (the correlation length). Within each blob, the segment is excited by thermal energy $\left(k_{\mathrm{B}} T\right)$ so that its center of gravity undergoes a Brownian motion. These thermal blobs have a similar size. In an athermal solvent, there is no interaction between them and the tube so that they experience a similar microenvironment, resulting in only one (fast) relaxation mode. In a less good or $\theta$ solvent, the segment-segment interaction near the entanglement points is expected to be stronger. Therefore, the blobs near each entanglement point should move slower than those in the middle between two entanglement points; namely, the blobs experience two different microenvironments, as schematically shown in Scheme 1 b. The increase of polymer concentration makes the tube thinner. Up to one point, the motions of the segments perpendicular to the tube near the entanglement point is so limited that the segments can only randomly oscillate in the tube. This might explain why its characteristic relaxation time becomes less dependent on or even independent of the scattering vector.

The temperature dependence studies also support our speculation about the nature of the slow mode. The decrease of the solution temperature makes the segment-segment interaction stronger so that each chain contracts. When $C / C_{\mathrm{e}}<1$, the chain contraction disentangles the transient clusters so that the slow mode becomes weak or even disappears at lower solution temperatures. However, when $C>C_{\mathrm{e}}$, the chain contraction cannot switch the solution from semidilute to dilute so that decreasing the solution temperature only increases the segment-segment interaction. This is why the slow mode becomes slower and more visible at lower temperatures.

\section{The slow mode in PNIPAM/ $\mathrm{H}_{2} \mathrm{O}$ system}

The solution dynamics in poly( $N$-isopropylacrylamide) (PNIPAM)/ $\mathrm{H}_{2} \mathrm{O}$ system was selected and examined by dynamic LLS because of its thermal-responsive properties, which exhibits a lower critical solution temperature around $32^{\circ} \mathrm{C}$. In such a way, similar to the PS in cyclohexane, we are able to use temperature dependence of the solvent-polymer interaction to investiagte effects of the shrinkage of polymer chains with increasing temperature, and consequently disentanglement of transient network, which in turn can prove the coexistence of individual chains and large transient network in semidilute solutions when $C / C_{\mathrm{e}}<1$.

For PNIPAM in semidilute aqueous solutions, Hirotsu and coworkers ${ }^{120}$ found that the correlation function measured by dynamic LLS could be fitted to single exponential quite well through the whole temperature range investigated $\left(25-40{ }^{\circ} \mathrm{C}\right)$. They claimed that the translational motion of polymer molecules is profoundly inhibited due to the relative immobility of densely packed coils and the intermolecular entanglement effect; the correlation function represents the relaxation of the internal or pseudogel mode. However, our recent result found that the single relaxation mode measured from dilute PNIPAM aqueous solution by dynamic LLS turns into one fast and one slow relaxation mode when the concentration enters into semidilute regime. Figure 10a shows typical plots of the intensity-intensity correlation function for the PNIPAM/ $\mathrm{H}_{2} \mathrm{O}$ system in both the dilute and semidilute regimes, and Figure 10b shows the corrsponding plots of characteristic relaxation time distribution function analyzed by the CONTIN method. When $C<C^{*}\left(C^{*}=1.9-2.8 \times 10^{-3} \mathrm{~g} \mathrm{ml}^{-1}\right.$ at $\left.25^{\circ} \mathrm{C}\right)$, a single relaxation mode is observed, which is related to the mutual diffusion of individual polymer chains. As the concentration increases
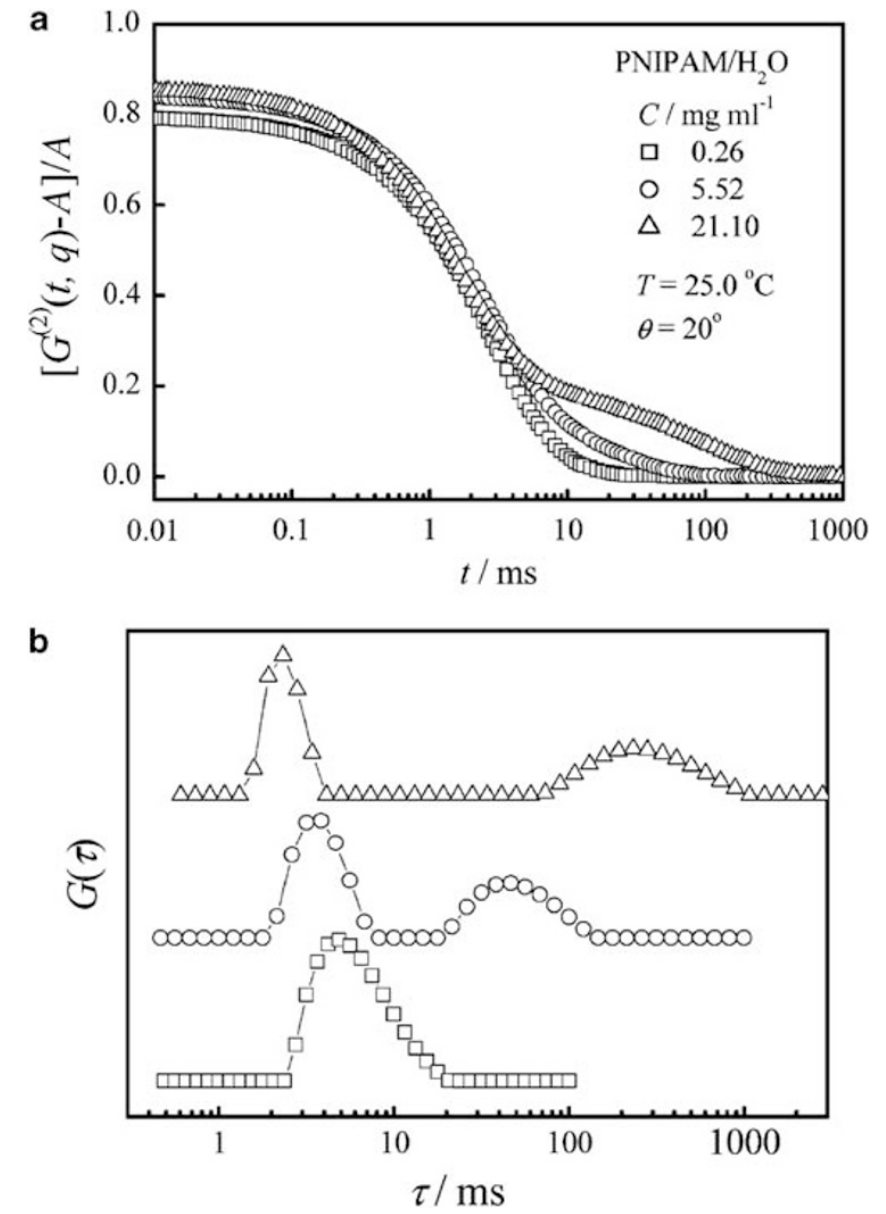

Figure 10 (a) Concentration dependence of intensity-intensity time correlation function $\left[G^{(2)}(t, q)-A\right] / A$ and $(\mathbf{b})$ their corresponding characteristic relaxation time distribution function $G(\tau)$ of PNIPAM/ $\mathrm{H}_{2} \mathrm{O}$ in both dilute and semidilute regimes. Reproduced with permission from Yuen et al. ${ }^{96}$ Copyright (2006) American Chemical Society.

and enters the semidilute regime, the chains begin to overlap and entangle, and new dynamical processes involving interchain interaction and disentanglement begin to occur besides the fast relaxation mode. The slow mode becomes more and more evident, and its characteristic relaxation time shifts toward slow direction with increasing concentrations.

Figure 11 shows the temperature dependence of characteristic relaxation time distribution function obtained from a semidilute solution. The data on the $x$ axis is shifted by $(T / \eta)$ so that the peak positions at different temperatures can be directly compared, where $\eta$ is the solvent viscosity at the absolute temperature. It is clear that the fast mode shifts to the slower direction, whereas the slow mode becomes faster with increasing temperatures when $T<30.0^{\circ} \mathrm{C}$. The effects are just contrary to that of increasing concentrations. The solvent quality tends to be poor with increasing temperature, which will result in fewer entanglement points and, consequently, a loose network with more defects or larger structural fluctuation. However, at $T=30.0^{\circ} \mathrm{C}$, phase separation happens. After keeping the solution at this temperature for $32 \mathrm{~h}$, the characteristic relaxation time distribution function turns out to be single narrow mode, which is $q^{2}$ dependent and related to collective diffusion of aggregates. The temperature dependence studied for PNIPAM $/ \mathrm{H}_{2} \mathrm{O}$ further supports 


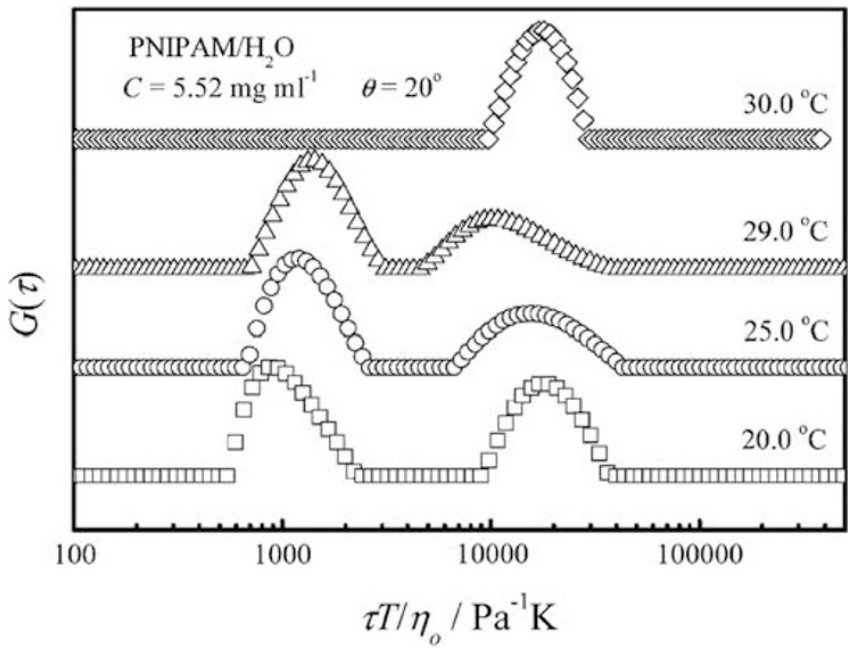

Figure 11 Temperature dependence of characteristic relaxation time distribution function $G(\tau)$ for a semidilute aqueous solution of PNIPAM. Reproduced with permission from Yuen et al. ${ }^{96}$ Copyright (2006) American Chemical Society.

our speculation about the nature of the slow mode that for $C / C_{\mathrm{e}}<1$, it is related some large structures.

\section{The slow mode in PEO-PPO-PEO/ $\mathrm{H}_{2} \mathrm{O}$ system}

Block copolymes normally consist of a few chemically distinct short chains joined by covalent bonds. In a solvent selectivley good only for one block, they can display more complicated structures and morphology, such as polymeric micelles, than homopolymers even in dilute solution. ${ }^{121,122}$ As for triblock copolymers in a selective solvent, the results becomes even more complicated because triblock chains can form flower-like micelles or interconnected clusters or even a gel network, depending on whether the solvent is selectively good for the middle or the two end blocks. In semidilute solution, because of the existence of overlapping and entanglement, new dynamical processes come into the additional problem. Therefore, in comparison with dynamics in homopolymers, the results of block copolymer chains in semidilute solution were more controversial and diversified. ${ }^{123-141}$

The system of poly(ethylene oxide)-poly(propylene oxide)-poly (ethylene oxide) (PEO-PPO-PEO) in $\mathrm{H}_{2} \mathrm{O}$ was also selected to investiage the solution dynamics because of the well-known fact that it can exhibit temperature-induced micellization phenomenon at a certain concentration range. Figure 12a shows typical plots of intensity-intensity correlation function for the PEO-PPO-PEO/ $\mathrm{H}_{2} \mathrm{O}$ system in both the dilute and semidilute regimes, and Figure $12 \mathrm{~b}$ shows the corresponding plots of characteristic relaxation time distribution analyzed by the CONTIN method. The apperant coherence is lower for dilute solution in Figure 12a due to its weak scattering intensity. The same tendency with that in the PNIPAM/ $\mathrm{H}_{2} \mathrm{O}$ system can be seen as increasing concentrations. Here, $T=20^{\circ} \mathrm{C}$ is below the critical micellization temperature of all the mentioned concentration according to Hatton et al. ${ }^{142}$ The slow mode is two orders of magnitude slower than the fast mode for PEO-PPO-PEO/ $\mathrm{H}_{2} \mathrm{O}$ at the concentration which is twice $C^{\star}$.

For nonionic polymeric surfactants PEO-PPO-PEO triblock copolymer in aqueous solutions, the dehydration gradually becomes significant with increasing temperature and consequently results in a decrease of the size of copolymer chains. When the temperature is above the critical micellization temperature of a certain concentration, micelles will form. Figure 13 shows the temperature dependence of
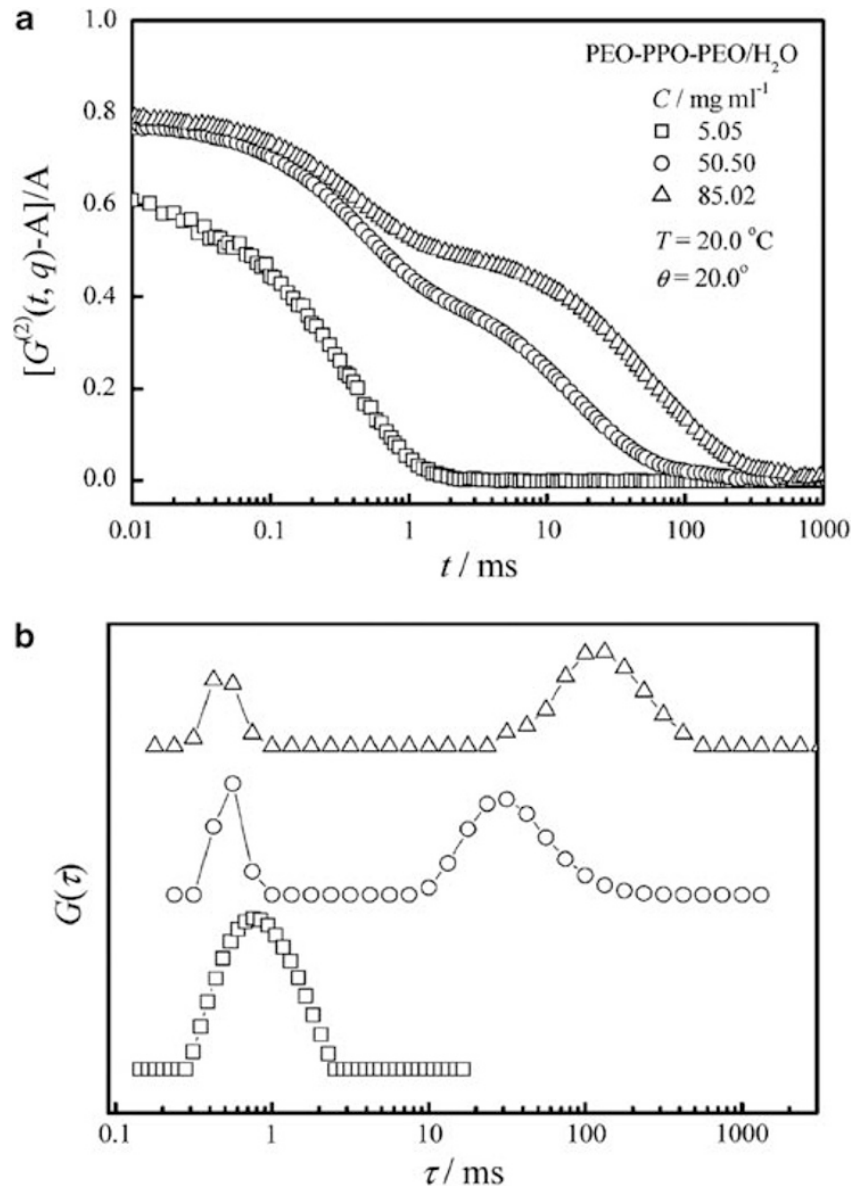

Figure 12 (a) Concentration dependence of intensity-intensity time correlation function $\left[G^{(2)}(t, q)-A\right] / A$ and (b) their corresponding characteristic relaxation time distribution function $G(\tau)$ of PEO-PPO-PEO/ $\mathrm{H}_{2} \mathrm{O}$ in both dilute and semidilute regimes. Reproduced with permission from Yuen et al. ${ }^{96}$ Copyright (2006) American Chemical Society.

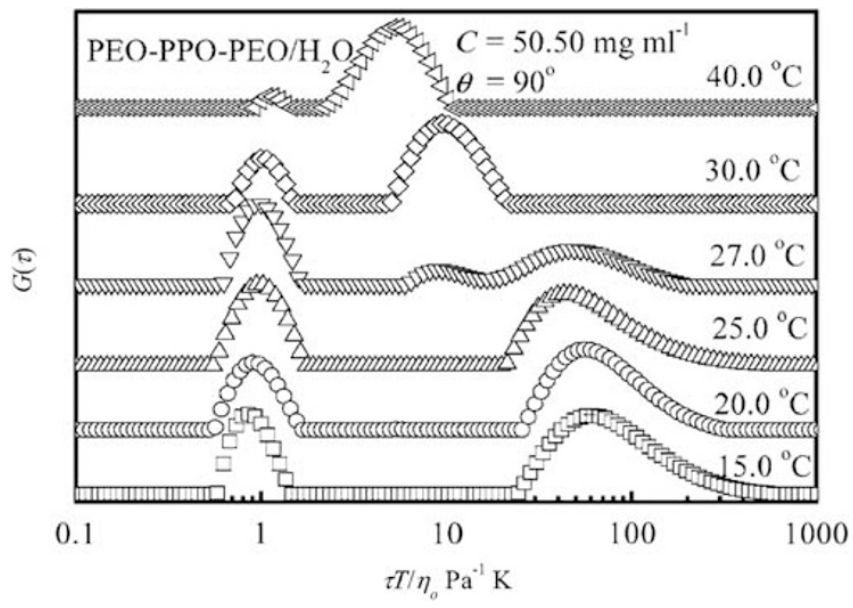

Figure 13 Temperature dependence of characteristic relaxation time distribution function $G(\tau)$ for a semidilute aqueous solution of PEO-PPOPEO. Reproduced with permission from Yuen et al. ${ }^{96}$ Copyright (2006) American Chemical Society.

characteristic relaxation time distribution function obtained from a semidilute solution. Below $25^{\circ} \mathrm{C}$, the same tendency of fast and slow relaxation mode changing with temperture was observed, compared 
with that in PNIPAM system. With increasing temperature, it is clear that an intermediate mode appears accompanied by the disappearance of the slow mode. The intermediate mode is evident at high temperature, and its relaxation rate is $q^{2}$ dependent; therefore it can be interpreted as collective diffusion of micelles.

In summary, the special thermal-sensitive properties of PNIPAM/ $\mathrm{H}_{2} \mathrm{O}$ and PEO-PPO-PEO/ $\mathrm{H}_{2} \mathrm{O}$ systems enable us to follow the temperature-induced shrinkage of polymer chains and, consequently, disentanglement of transient network in semidilute solutions formed by overlapped or entangled polymer chains, without changing the overall concentrations. The difference of the nature of slow mode between these two systems is that aggregates are formed in the PNIPAM $/ \mathrm{H}_{2} \mathrm{O}$ system but micelles are formed in the PEO-PPO$\mathrm{PEO} / \mathrm{H}_{2} \mathrm{O}$ systems when the solvent becomes poor.

\section{Effect of crosslinking on dynamics during the sol-gel transition}

Up to this point, our results clearly show that the slow relaxation observed in the semidilute solutions is real, not because of previously suggested artifacts in the solution preparation. The concentration and temperature dependence of the characteristic relaxation time of slow mode in different systems also suggest that such a slow mode has two different natures. When $C$ is lower than the entanglement concentration $\left(C_{\mathrm{e}}\right)$, it can be related either to the large temporal clusters formed by some entangled chains. However, at higher entanglement concentration, the restricted interaction of polymer chains near the entanglement points likely results in the slow mode. If this is the case, one may wonder that in situ crosslinking of polymer chains in a semidilute solution can artificially increase the correlation between different entanglement points, consequently increasing the relative contribution of the slow mode as crosslinking proceeded. ${ }^{101-103}$

To prove that, we synthesized a linear poly(methyl methacrylateco-7-acryloyloxy-4-methylcoumarin) (P(MMA-co-AMC)) copolymer and studied the dynamics of such a copolymer in semidilute solutions by dynamic LLS. The advantage of using such a system is relied on the fact that two coumarin groups can undergo a $(2+2)$ photodimerization when irradiated with an ultraviolet light $(310 \mathrm{~nm})$ as shown in Scheme 2. Such a dimerization reaction can be used to bind the copolymer chains together in chloroform in a completely controllable manner so that we are able to investigate the effect of crosslinking on the fast and slow relaxation modes of semidilute solutions at each photo-reaction stage of the sol-gel transition.

Figures 14 shows that in the initial semidilute solution before the photo-crosslinking, $g^{(2)}(q, \tau)$ has two distinct relaxation modes. The fast relaxation corresponds to the cooperative diffusion of the subchains between two entangled points, which is well described by the 'blob' or scaling theory, ${ }^{116,117}$ whereas the controversial slow relaxation can be attributed to the relatively stronger segment-segment interaction near the entanglement points makes the congested polymer chain nonuniform..$^{99,102,103}$ Previously, we also speculated that such slow mode might be related to some long-range density fluctuation. ${ }^{102}$ As the crosslinking proceeds, the slow relaxation becomes even slower, and at the same time, its contribution to the total scattering intensity increases, reflecting in the raise of its amplitude. It clearly shows that the crosslinking enhances the restricted movement of the subchains near the entanglement points, resulting in the slow mode. The flowing

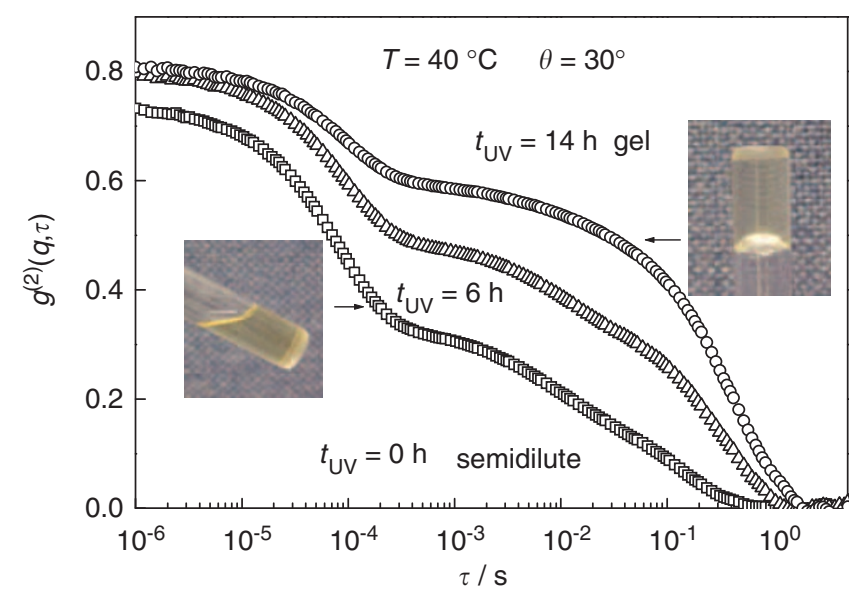

Figure 14 Normalized intensity-intensity time correlation functions of crosslinkable P(MMA-co-AMC) chains in chloroform during the sol-gel transition. Reproduced with permission from Ngai et al. ${ }^{102}$ Copyright (2004) American Chemical Society.<smiles>COC(=O)C(C)(I)CI(C)C(=O)Oc1ccc2c(C)cc(=O)oc2c1</smiles>

$\mathrm{P}$ (MMA-co-AMC)

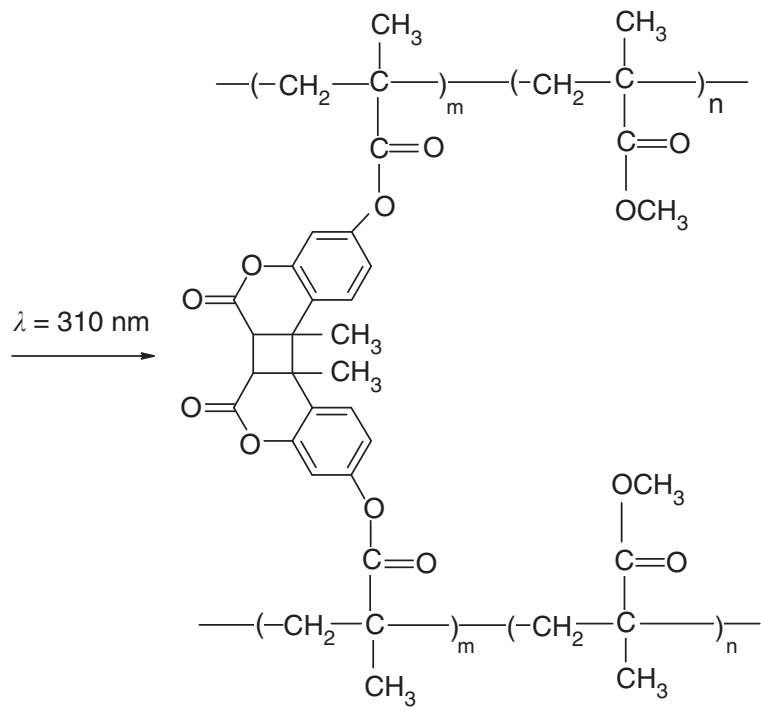

Scheme 2 Photodimerization-induced crosslinking of P(MMA-co-AMC) copolymer chains in chloroform. Reproduced with permission from Ngai et al. ${ }^{103}$ Copyright (2003) American Chemical Society. 


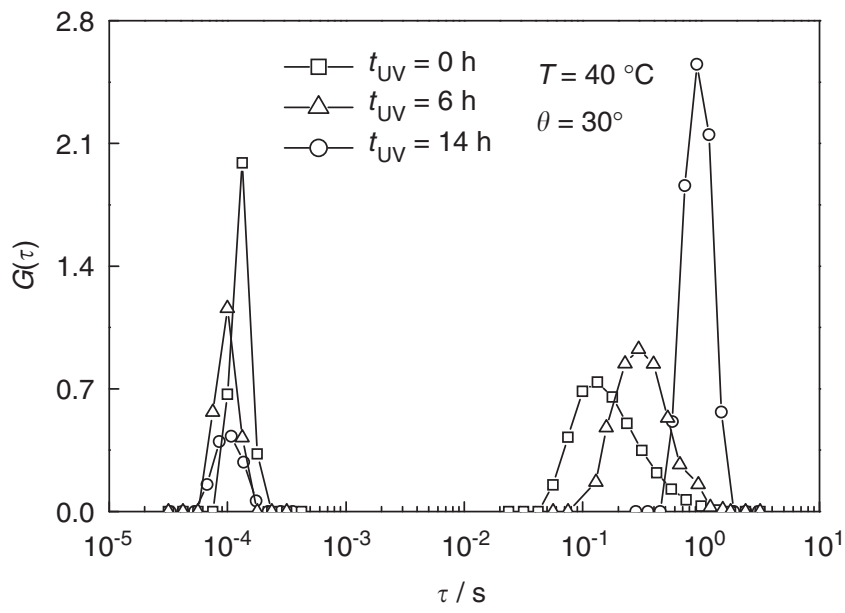

Figure 15 Peak area-normalized intensity distribution of characteristic decay time $f(\tau)$ of crosslinkable $P(M M A-c o-A M C)$ chains in chloroform during the sol-gel transition, where $f(\tau)$ is $\tau$-weighted $G(\tau)$, that is, $f(\tau) \equiv \tau G(\tau)$, because the $x$ axis is logarithmic. Reproduced with permission from Ngai et al. ${ }^{102}$ Copyright (2004) American Chemical Society.

semidilute solution finally becomes a macroscopically immobile chemical gel after $\sim 9 \mathrm{~h}$ ultraviolet irradiation, as shown in the insets. Note that even in the gel state, $g^{(2)}(q, \tau)$ can still completely relax to zero even the when relaxation is fairly slow, indicating that there is no static frozen-in component inside. It is important to note that the complete relaxation of $g^{(2)}(q, \tau)$ is not due to an improper normalization. ${ }^{143,144}$ This is because here the intercept of $g^{(2)}(q, \tau)$ at $\tau \rightarrow 0$ increases during the sol-gel transition. The lack of static frozen-in components is supported by the sample-position-independent scattering intensity; for example, no speckle was detected when we randomly turned the LLS cell to let the laser hit different parts of the gel.

Figure 15 shows the variation of $G(\tau)$ from a semidilute solution to a gel network, where $\tau=1 / \Gamma$. During the sol-gel transition, the bimode distribution is clearly preserved, although the relative contribution of the fast relaxation decreases. Shibayama and colleagues ${ }^{145}$ also reported that the fast mode remained in the formation of silica gels. Another interesting point is that the position of the peak related to the fast relaxation nearly remains during the sol-gel transition, different from other gelling systems. ${ }^{127,146}$ The peak related to the slow relaxation progressively shifts to a longer delay time, that is, the crosslinking hinders motions of interacting polymer chains. In other words, the motion of the segments near the entanglement point (or crosslinked point) is so limited that the segments may only randomly oscillate. It should be addressed that the crosslinking mostly occurs at the entangled points because only two very nearby coumarin groups can undergo the $(2+2)$ photocycloaddition. Therefore, the crosslinking should have a limited effect on the length of the subchains ('blobs'). This explains why the peak related to the fast relaxation remains its position.

Figure 16 shows the effect of temperature on the intensity weighting (relative contribution) of fast relaxation $\left(A_{\mathrm{f}}\right)$ during the sol-gel transition. It indicates that the intensity contribution of the $A_{\mathrm{f}}$ increases with the temperature, but the trend is quite different with and without the crosslinking. Note that $A_{\mathrm{f}}+A_{\mathrm{s}}=1$ so that we only plot $A_{\mathrm{f}}$ vs $T$. The fast relaxation of the crosslinked chains contributes very little at lower temperatures. It should be noted that the overall timeaveraged scattering intensity $\left(\langle I\rangle_{\mathrm{T}}\right)$ measured in static LLS is the sum of $\langle I\rangle_{\text {fast }}$ and $\langle I\rangle_{\text {slow }}$, and $\langle I\rangle_{\mathrm{T}}$ increases $\sim 180$ times as the

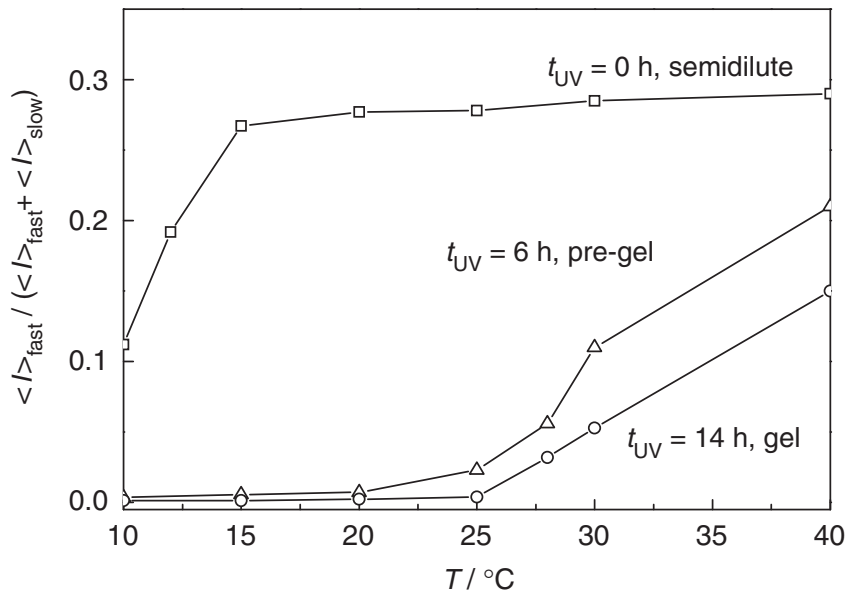

Figure 16 Temperature dependence of intensity weighting (relative contribution) of fast relaxation $\left(A_{\mathrm{f}}\right)$ of crosslinkable P(MMA-co-AMC) chains in chloroform during the sol-gel transition. Note that $A_{\mathrm{f}}+A_{\mathrm{s}}=1$. Reproduced with permission from Ngai et al. ${ }^{102}$ Copyright (2004) American Chemical Society.

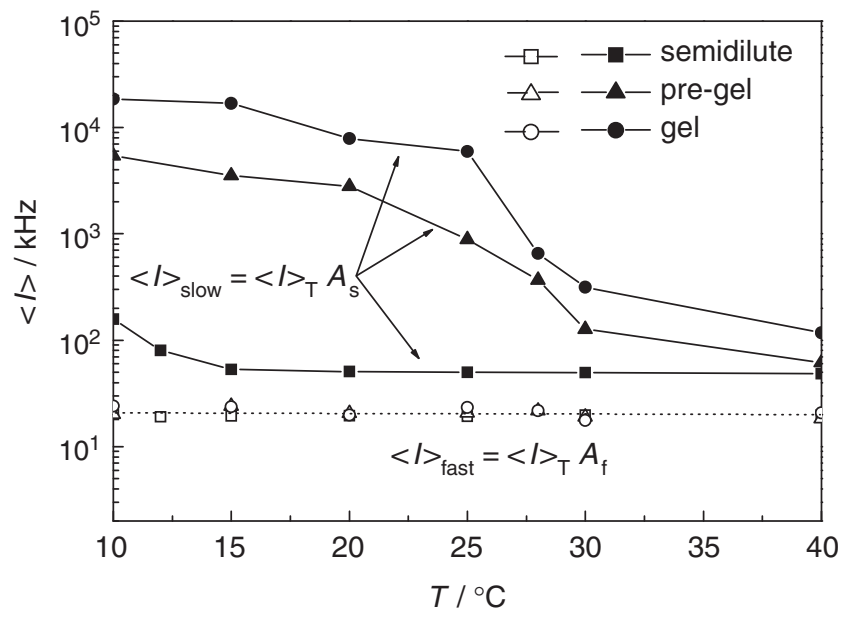

Figure 17 Temperature dependence of time-averaged scattering intensities $\left(\langle I\rangle_{\text {fast }}\right.$ and $\left.\langle I\rangle_{\text {slow }}\right)$ related to fast and slow relaxation of crosslinkable $\mathrm{P}(\mathrm{MMA}-\mathrm{Co}-\mathrm{AMC})$ chains in chloroform during the sol-gel transition, where $\langle I\rangle_{\mathrm{T}}$ is overall time-averaged scattering intensity measured in static LLS. Reproduced with permission from Ngai et al. ${ }^{102}$ Copyright (2004) American Chemical Society.

temperature decreases. Therefore, it is necessary and right to calculate the absolute average scattering intensities $\left(\langle I\rangle_{\text {fast }}\right.$ and $\left.\langle I\rangle_{\text {slow }}\right)$, respectively, for the fast and slow modes, instead of $A_{\mathrm{f}}$ and $A_{\mathrm{s}}$. The results are shown in Figure 17. It is helpful to note that static and dynamic LLS measurements were carried out in a back-to-back manner on the same sample position under identical conditions and at the same $q$. On the basis of Figures 16 and 17, we know that the decrease of $A_{\mathrm{f}}$ is attributed to the increase of $\langle I\rangle_{\text {slow }}$. This is understandable because the fast relaxation is related to the motions of the subchains (blobs) between two entangled (crosslinked) points and the temperature should have no effect on the average length of the subchains and the refractive index contrast between the blobs and solvent, as the temperature dependence of the refractive index $(\mathrm{d} n / \mathrm{d} T)$ is only in the order of $10^{-4}$. 
As for the increase of $\langle I\rangle_{\text {slow }}$ we should note that in semidilute solution polymer, chains are highly entangled with each other. Inside the volume of one chain, there also exist many other chains, especially when the chain is long. Assuming that each chain with a radius of gyration $\left(R_{\mathrm{g}}\right)$ has $n_{\mathrm{s}}$ segments and there are $n_{\mathrm{p}}$ chains inside its occupied space $\left(\sim R_{\mathrm{g}}\right)^{3}$, the scattering intensity $(\langle I\rangle)$ from the $n_{\mathrm{s}} n_{\mathrm{p}}$ segments on the basis of the LLS theory is related the position of each segment $(r)$ as follows, ${ }^{108}$

$$
\langle I\rangle_{\mathrm{T}} \propto\left\langle\sum_{j=1}^{n_{\mathrm{s}} n_{\mathrm{p}}} \sum_{k=1}^{\mathrm{n}_{\mathrm{s}} \mathrm{n}_{\mathrm{p}}} \operatorname{expi}\left[\overrightarrow{\mathrm{q}} \cdot\left(\overrightarrow{\mathrm{r}_{\mathrm{j}}}-\overrightarrow{\mathrm{r}_{\mathrm{k}}}\right)\right]\right\rangle
$$

If the motions of $n_{\mathrm{s}} n_{\mathrm{p}}$ segments are fully correlated, $\langle I\rangle_{\mathrm{T}} \propto\left(n_{\mathrm{s}} n_{\mathrm{p}}\right)^{2}$ when $q R_{\mathrm{g}} \ll 1$; although if the motions of $n_{\mathrm{s}} n_{\mathrm{p}}$ segments are completely independent, $\langle I\rangle_{\mathrm{T}} \propto n_{\mathrm{s}} n_{\mathrm{p}}$. In reality, $\langle I\rangle_{\mathrm{T}}$ is between $n_{\mathrm{s}} n_{\mathrm{p}}$ and $\left(n_{\mathrm{s}} n_{\mathrm{p}}\right)^{2}$ in semidilute solution because the segments between different chains are only partially correlated. The crosslinking makes the segments between different chains more correlated so that the scattering intensity increases. On the other hand, the increase of the solution viscosity at lower temperatures enhances the hydrodynamic interaction of the segments between different chains. This is why $\langle I\rangle_{\text {slow }}$ increases with a decreasing temperature.

\section{The slow mode in salt-free or low-salt polyelectrolytes solution}

Dynamics of polyelectrolytes in salt-free or low-salt solutions has attracted much attention after Lin et al. ${ }^{111}$ reported their dynamic LLS observation of an extremely slow diffusive mode in the low-salt poly(L-lysine) solutions. Later, it was shown that there are actually a fast and a slow relaxation mode in dynamic LLS measurements of saltfree or low-salt dilute polyelectrolyte solution in comparison with the translational diffusive relaxation mode of individual neutral chains with similar lengths. Some reported slow relaxation modes were really slow with a hydrodynamic size of $10^{2}-10^{3} \mathrm{~nm}$ or larger. Gradually, such two modes have been reported for nearly all charged macromolecules, including both synthetic and biological polyelectrolytes in aqueous and nonaqueous solutions. ${ }^{76-84}$ Therefore, these two modes are general features of salt-free or low-salt polyelectrolyte solutions.

In the past, the fast mode in polymer dilute and semidilute solutions has been attributed to different origins, such as propagation of excitations in a polyelectrolyte pseudolattice or the free diffusion of the noncaged chains/particles. When polymer chains are charged in a saltfree or low-salt solution, their translational diffusive relaxation modes split into a fast and a slow diffusive relaxation mode. Muthukumar ${ }^{86-88}$ and Lee and colleagues ${ }^{111}$ suggested that the fast mode, independent of the chain length, would be related to the coupled diffusion $\left(D_{\mathrm{f}}\right)$ of polyelectrolyte chains and their small counterions, namely, in a salt-free or low-salt solution; the counterions must be condensed nearby the chain backbone, and the fluctuation of condensed counterions toward and away from each chain backbone induces an electric field that leads to an electrophoresis-mobility-related diffusive relaxation.

On the other hand, the interpretation of the slow mode, especially for those very slow relaxation modes observed in salt-free or low-salt polyelectrolyte solutions, is even more controversial. It has been attributed to dynamics of large multichain domains (transient aggregates or clusters) formed due to electrostatic interaction ${ }^{76-80}$ or some insoluble chain clusters, or even a trace amount of large particles introduced during the imperfect preparation of solution. ${ }^{89-93}$ The transient cluster interpretation was supported by studies of poly(styrenesulfonate) with sodium counterions in aqueous solutions under dialysis. For a long time, this transient cluster has been attributed to the effective interaction between similarly charged segments, namely, the overlapping of the ion clouds of neighboring polyions, so that the more loosely associated small ions are 'shared' by two or more polyions chains, resulting in a fluctuating dipole field that tends to retard the relative motions of those participating polyions.

Experimentally, a polymer solution is normally prepared by allowing a macroscopic piece of polymer to dissolve in a solvent. The so-called permanent chain clusters must be from the incomplete dissolution in the solution preparation, not the chain association, because we do not place individual chains into a solvent. Cong et al. ${ }^{95}$ addressed those possible problems and recently prepared a 'virgin' poly(styrenesulfonate) sample directly from 4-styrene-sulfonic sodium salt in an aqueous medium. They then used dialysis to in situ change the salt concentration. Even in that case, the solution cleanliness still deteriorates after the dialysis. To avoid some possible problems in sample preparation, ideally, one would like to have a polymer solution in which polymer chains can be in situ switched from a completely neutral to a fully charged state by some chemical reactions under a mild condition. We have searched such a system for a long time. Recently, using the Staudinger ligation to attach 4 -(5'-(1-(dimethylamino)-ethylidene-amino)pentyl)-1-methyl-2-(diphenylphosphino)terephthalate to poly( $p$-azidomethylstyrene)-co-polystyrene, we successfully prepared a novel polymer, poly(4-(4-((5-(1-(dime-thylamino)ethylideneamino)pentyl-oxy)carbonyl)-2-(diphenylphosphoryl)benzoyloxymethyl-styrene)-co-poly(styrene) ( $\mathrm{P}($ 'amidine'MS)-co-PS), which undergo a reversible neutral-charged-neutral transition when $\mathrm{CO}_{2}$ and $\mathrm{N}_{2}$ are alternatively bubbled through its $\mathrm{N}, \mathrm{N}$-dimethylformamide (DMF) solution as illustrated in Scheme 3. Armed with this novel polymer, we are able to rule out any interference of impurities or permanent chains clusters on solutions, and in situ study the variation of solution dynamics of polymer chains during the neutral-chargedneutral transition.
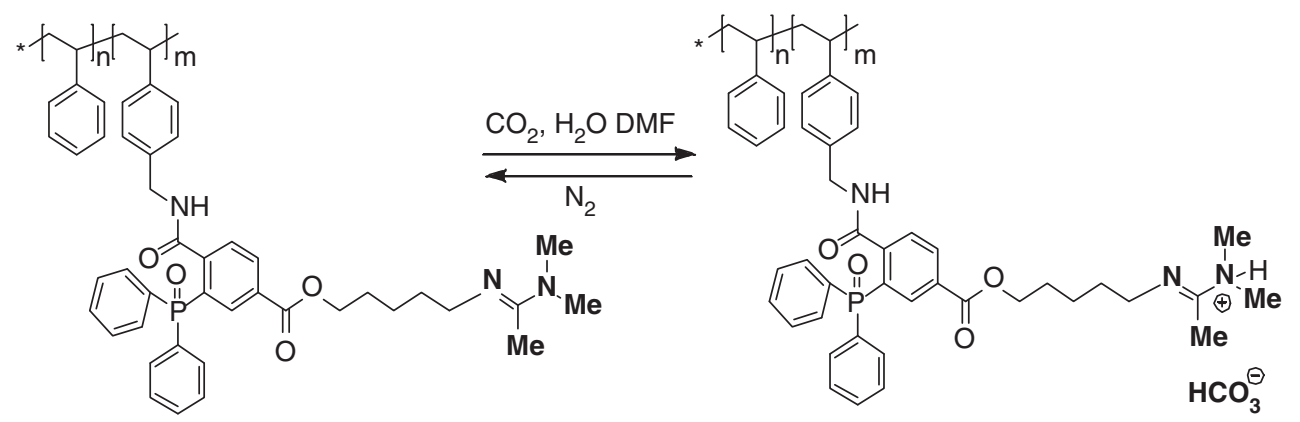

Scheme 3 Reversible change between P('amidine'MS)-co-PS and its charged state in DMF with $0.5 \% \mathrm{H}_{2} \mathrm{O}$ after alternating bubbling of $\mathrm{CO}_{2}$ and $\mathrm{N}_{2}$. Reproduced with permission from Zhou et al. ${ }^{107}$ Copyright (2009) American Chemical Society. 


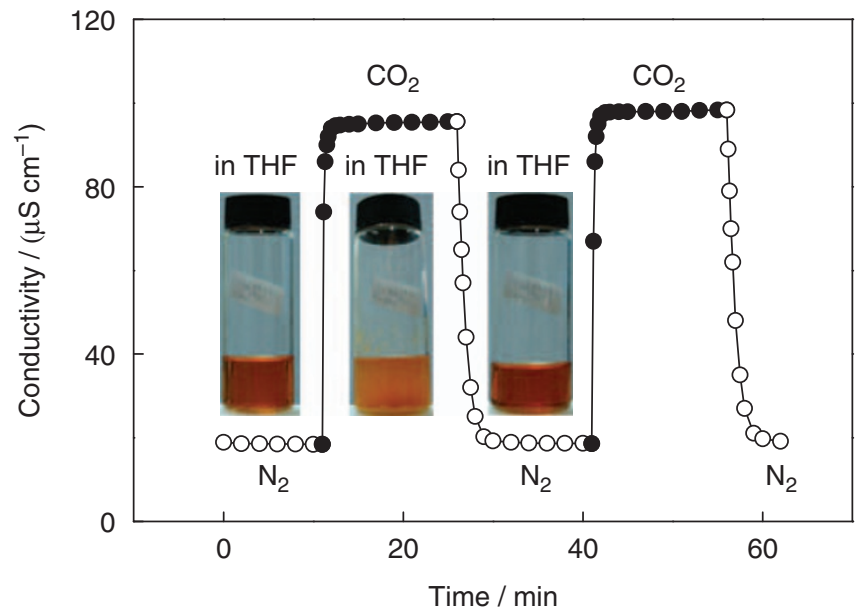

Figure 18 Conductivity of $\mathrm{P}$ ('amidine'MS)-co-PS in DMF with $0.5 \% \mathrm{H}_{2} \mathrm{O}$ at $25^{\circ} \mathrm{C}$ after alternative bubbling of $\mathrm{CO}_{2}$ and $\mathrm{N}_{2}$, where polymer concentration is $7.3 \mathrm{mg} \mathrm{ml}^{-1}$. Insets are photos of $\mathrm{P}$ ('amidine'MS)-co-PS in THF with $0.5 \%$ $\mathrm{H}_{2} \mathrm{O}$ in different states before and after $\mathrm{CO}_{2}$ and $\mathrm{N}_{2}$ bubbling, where polymer concentration is $25.0 \mathrm{mg} \mathrm{ml}^{-1}$. Reproduced with permission from Zhou et al. ${ }^{107}$ Copyright (2009) American Chemical Society.

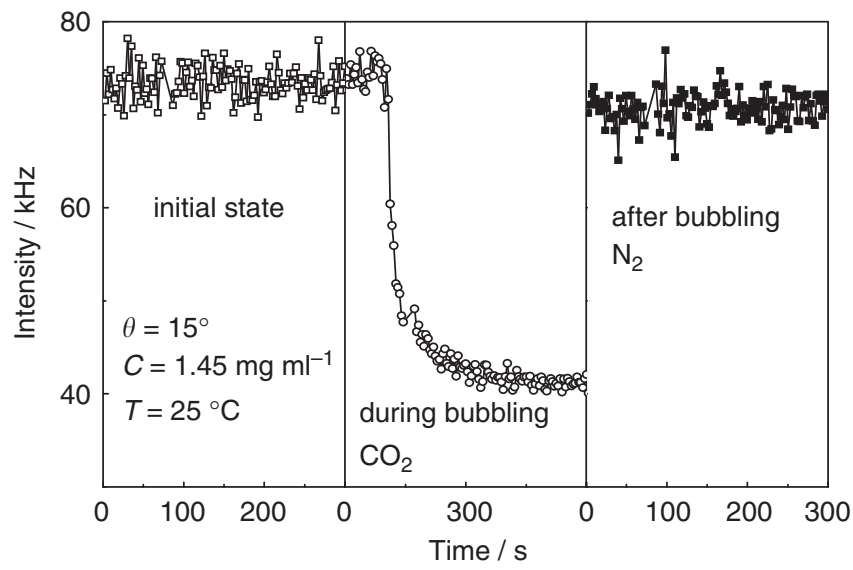

Figure 19 Time dependence of time-average scattering intensity of $\mathrm{P}$ ('amidine'MS)-co-PS in DMF with $0.5 \% \mathrm{H}_{2} \mathrm{O}$ at different states before and after $\mathrm{CO}_{2}$ and $\mathrm{N}_{2}$ bubbling. Reproduced with permission from Zhou et al. ${ }^{107}$ Copyright (2009) American Chemical Society.

Figure 18 shows that after $\mathrm{CO}_{2}$ and $\mathrm{N}_{2}$ gases were alternatively bubbled $30 \mathrm{~min}$ through each solution of $\mathrm{P}$ ('amidine'MS)-co-PS in DMF at $25^{\circ} \mathrm{C}$, the solution conductivity dramatically changes five times and switches between two constant values. The switching is completely reversible. The inset photos in Figure 18 are the appearance of the solution of $\mathrm{P}$ ('amidine'MS)-co-PS in a different solvent (tetrahydrofuran, THF) with $0.5 \% \mathrm{H}_{2} \mathrm{O}$, in which the charged $\mathrm{P}$ ('amidine'MS)-co-PS chains have an extremely low solubility. The change solution appearance from clear to cloudy and from cloudy to clear during the alternative bubbling of $\mathrm{CO}_{2}$ and $\mathrm{N}_{2}$ clearly shows the expected neutral-charged-neutral transition of $\mathrm{P}$ ('amidine'MS)-co-PS chains in THF.

Dynamic LLS was also used to follow such neutral-charged-neutral transition of $\mathrm{P}$ ('amidine'MS)-co-PS chains. Figure 19 shows that as individual $\mathrm{P}$ ('amidine'MS)-co-PS chains are gradually charged during the $\mathrm{CO}_{2}$ bubbling, the time-average scattering intensity $(\langle I\rangle)$ decreases $\sim 45 \%$ over a period of $\sim 500 \mathrm{~s}$. The alternative bubbling of $\mathrm{CO}_{2}$ or $\mathrm{N}_{2}$ can reversibly switch $\langle I\rangle$ between two average values.

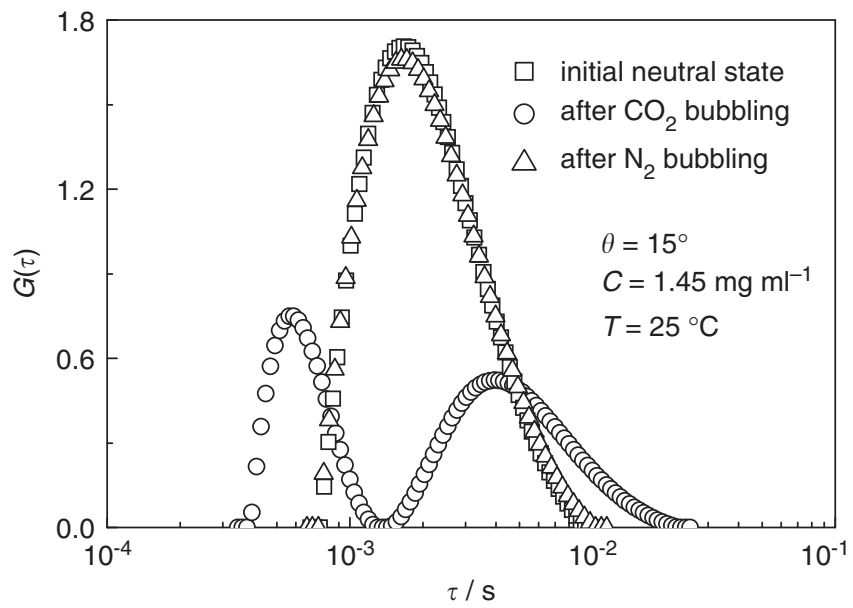

Figure 20 Characteristic relaxation time distributions in one cycle of $\mathrm{CO}_{2}$ and $\mathrm{N}_{2}$ bubbling of $\mathrm{P}$ ('amidine'MS)-co-PS solution. Reproduced with permission from Zhou et al. ${ }^{107}$ Copyright (2009) American Chemical Society.

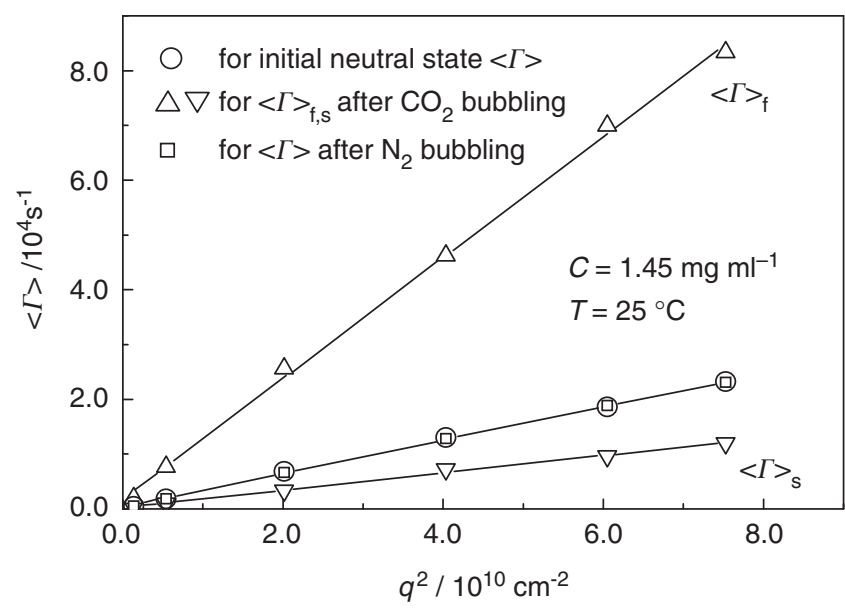

Figure 21 The scattering vector $(q)$ dependence of average characteristic line widths $(\Gamma)$ in one cycle of $\mathrm{CO}_{2}$ and $\mathrm{N}_{2}$ bubbling of $\mathrm{P}$ ('amidine'MS)co-PS solution. Reproduced with permission from Zhou et al. ${ }^{107}$ Copyright (2009) American Chemical Society.

It has been well known in LLS that $\langle I\rangle$ is proportional to $(\partial C / \partial \pi)_{\mathrm{T}}$, where $C$ and $\pi$ are polymer concentration and solution osmotic pressure, respectively. When polymer chains are charged, it is more difficult to induce the concentration fluctuation for a given osmotic pressure change because of electrostatic repulsion. Therefore, such a decrease of $\langle I\rangle$ after the chains are charged is understood.

Figure 20 shows variation of the characteristic relaxation time distribution $G(\tau)$ in one cycle of $\mathrm{CO}_{2}$ and $\mathrm{N}_{2}$ bubbling of the $\mathrm{P}$ ('amidine'MS)-co-PS solution. When the polymer chains are their initial neutral state before the $\mathrm{CO}_{2}$ bubbling, there is only one relaxation mode. When each $\mathrm{P}$ ('amidine'MS)-co-PS chain is charged after the $\mathrm{CO}_{2}$ bubbling, $G(\tau)$ splits into two peaks. The $\mathrm{N}_{2}$ bubbling can return $G(\tau)$ to its initial state, further indicating that the neutralcharged-neutral transition is completely reversible. The scattering vector $(q)$ dependence of average characteristic line widths $(\langle\Gamma\rangle)$ in the corresponding one cycle of $\mathrm{CO}_{2}$ and $\mathrm{N}_{2}$ bubbling of the $\mathrm{P}$ ('amidine'MS)-co-PS solution is shown in Figure 21. No matter whether the chains are charged, the average characteristic line width 


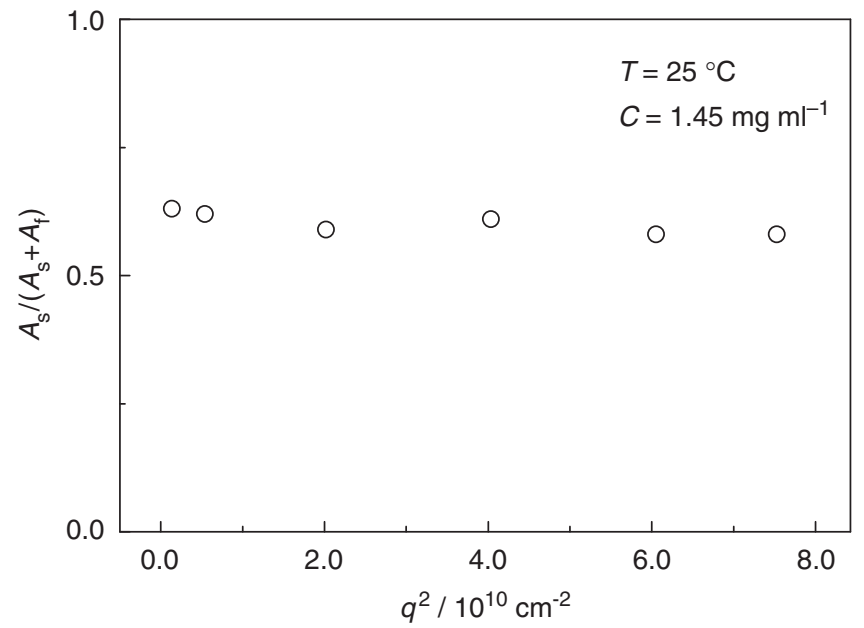

Figure 22 Scattering vector $(q)$ dependence of intensity contribution of slow relaxation mode $\left(A_{\mathrm{s}}\right)$ after $\mathrm{N}_{2}$ bubbling of $\mathrm{P}$ ('amidine'MS)-co-PS solution. Note that $A_{\mathrm{s}}+A_{\mathrm{s}}=1$. Reproduced with permission from Zhou et al. ${ }^{107}$ Copyright (2009) American Chemical Society.

$(\langle\Gamma\rangle=1 /\langle\tau\rangle)$ is a linear function of $q^{2}$, passing through the origin, indicating that these relaxation modes are diffusive. Further, Figure 22 shows that the intensity contribution of the slow relaxation mode $\left(A_{\mathrm{s}}\right)$ nearly remains a constant at different scattering angles, where $A_{\mathrm{f}}+A_{\mathrm{s}}=1$. The $q$ independence of the scattering intensity reveals that the slow mode is not related to scattering objects larger than $1 / q$, or $\sim 35-190 \mathrm{~nm}$.

These results are similar to those recently observed by Russo and colleagues. ${ }^{95}$ but they still attributed the slow mode to some temporal chain aggregates. We found that our results agree well with the interpretation of dynamics of individual polyelectrolyte chains in salt-free dilute solutions proposed by Muthukumar. ${ }^{86-88}$ Pusey and Tough ${ }^{147}$ attributed the fast and slow modes to the mutual and selfdiffusion, respectively. For example, in a short time, each chain moves over a short distance and the diffusion of counterions makes the charged particles to diffuse faster. After the relaxation of such 'collective diffusion', the 'self-diffusion' of individual chains in a long time or over a long length must be retarded by those surrounding interacting particles and becomes detectable in LLS. Our fast mode agrees well with the interpretation of the coupled diffusion of individual polyelectrolyte chains and their counterions in salt-free dilute solutions proposed by Lin et al. ${ }^{111}$ and Muthukumar, ${ }^{86-88}$ whereas the slow mode is related to the self-diffusion of the centerof-mass of individual chains under long-range electrostatic interaction-induced constrains of other surrounding chains, just like a cage, because our results reveal that the slow mode is related to small but slowly moving subjects, in other words, the long-range electrostatic interaction may slow down the self-diffusion of the center-of-mass of individual chain.

Figure 23 shows how $G(\tau)$ changes in the process of backing to the neutral state when $\mathrm{N}_{2}$ is slowly bubbled through the solution. It is clear that during the charge-to-neutral transition, $\langle\tau\rangle_{\text {fast }}$ increases, that is, $D_{\text {fast }}$ decreases, so that the coupled fast relaxation slows down. At the same time, the total scattering intensity $\langle I\rangle$ and the contribution from the slow relaxation $\left(A_{\mathrm{s}}\right)$ increase because the electrostatic interaction constrained the motion of chains weakly and the intensity of the fast mode, which is contributed to the coupled diffusive relaxation of individual polyelectrolyte chains and their counterions, diminishes as each chain becomes neutral.

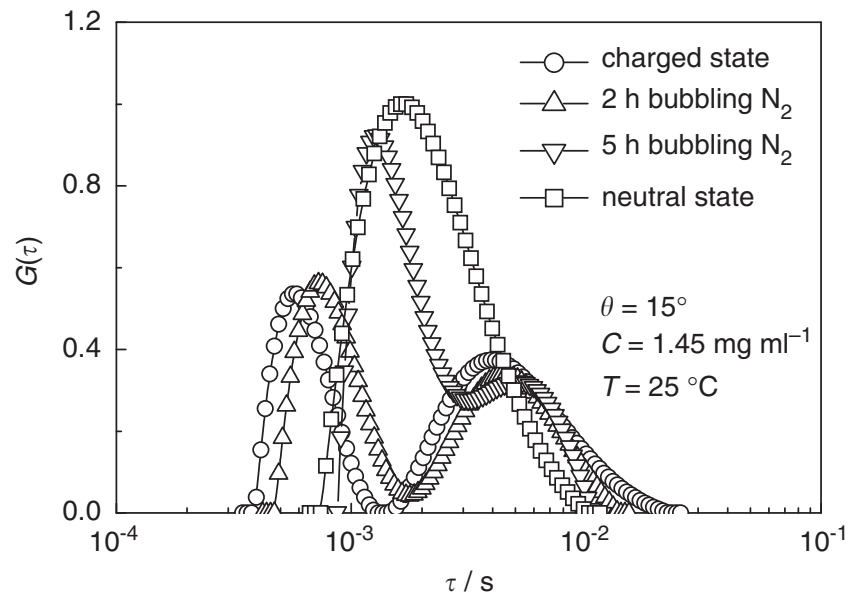

Figure 23 Characteristic relaxation time distributions $G(\tau)$ during the charge-to-neutral transition induced by slowly bubbling $\mathrm{N}_{2}$ through P('amidine'MS)-co-PS solution. Reproduced with permission from Zhou et al. ${ }^{107}$ Copyright (2009) American Chemical Society.

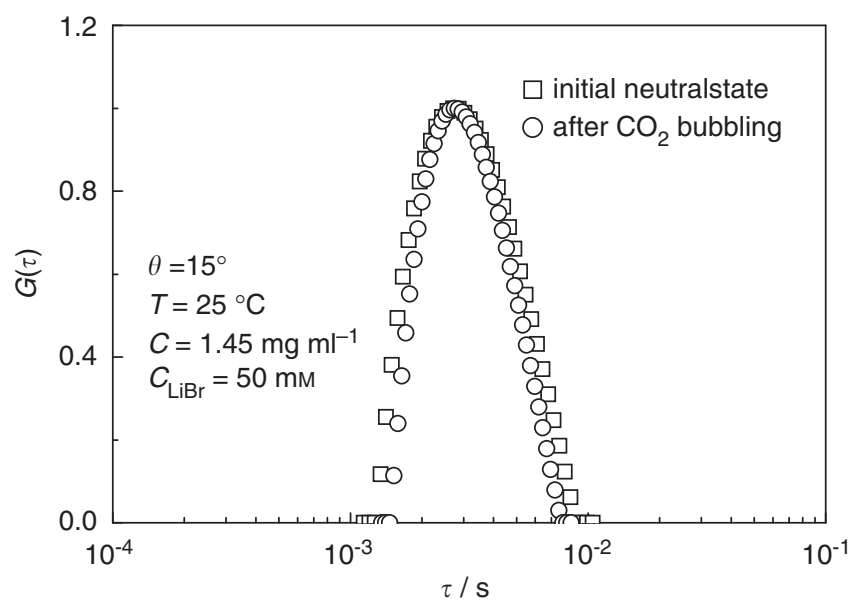

Figure 24 Characteristic relaxation time distributions $G(\tau)$ of $\mathrm{P}$ ('amidine'MS)-co-PS solution in the presence of $50 \mathrm{~mm} \mathrm{LiBr}$ before and after $\mathrm{CO}_{2}$ bubbling. Reproduced with permission from Zhou et al. ${ }^{107}$ Copyright (2009) American Chemical Society.

When the high amount of salt $\mathrm{LiBr}$ was added into the solution, as expected, we can find that there is only one relaxation mode after the $\mathrm{CO}_{2}$ bubbling. Figure 24 shows that the $\mathrm{CO}_{2}$ bubbling has no effect on $G(\tau)$ of charged $\mathrm{P}$ ('amidine'MS)-co-PS chains in dilute solutions; namely, the addition of $50 \mathrm{~mm} \mathrm{LiBr}$ sufficiently suppress electrostatic interaction among charges on the chain backbone so the charged chains behave like in the initial neutral state. The slightly larger $\left\langle R_{\mathrm{h}}\right\rangle$ is attributed to the chain swelling because the addition of LiBr makes DMF a better solvent.

In summary, by using a novel $\mathrm{P}$ ('amidine'MS)-co-PS that can be in situ and reversibly switched between a neutral state to a charged state by alternative bubbling of $\mathrm{CO}_{2}$ and $\mathrm{N}_{2}$, our LLS results reconfirm that when individual polymer chains are charged in salt-free dilute solutions, their translational diffusive relaxation in the neutral state splits into a fast and a slow diffusive mode due to long-range electrostatic interaction. As expected, adding high amount of salt can suppress the electrostatic interaction and the charged chains behave like the neutral ones with only one translational diffusive relaxation mode. The fast 


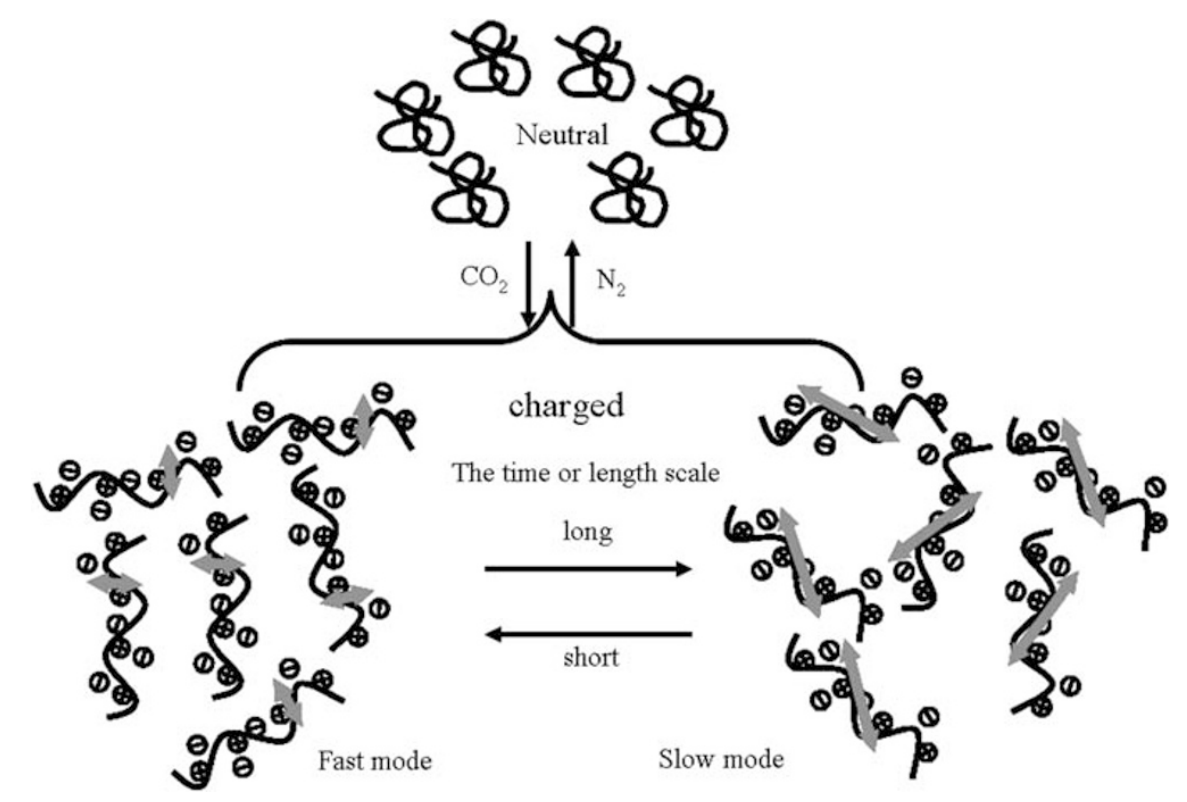

Scheme 4 Schematic of fast and slow relaxation modes after each P('amidine' MS)-co-PS chain becomes charged after the $\mathrm{CO}_{2}$ bubbling; namely, the fast mode is because of the coupling of the diffusion of the chain and the wiggling of chain segments induced by fluctuation of counterions condensed nearby the charged chain backbone in a salt-free or low-salt solution and the slow mode to the self-diffusion of the charged chain with an extended conformation. Reproduced with permission from Zhou et al. ${ }^{107}$ Copyright (2009) American Chemical Society.

mode can be attributed to the coupled diffusion originated from a convective current generated by an induced electric field arising from fluctuation of all charged species (charges on the chain and counterions) in the solution in a short time or length scale, whereas the slow mode is related to slowly moving scattering objects but it is not large, supporting the assumption of the retarded self-diffusion as Scheme 4 .

\section{Conclusion}

Using in situ anionic polymerization to directly prepare macroscopically homogeneous dust-free semidilute/concentrated PS solutions inside a LLS cell, we comparatively reexamined solution dynamics of PS in an athermal solvent (toluene) and in a less good/ $\theta$ solvent (cyclohexane in the range $34-50{ }^{\circ} \mathrm{C}$ ) by a combination of static and dynamic LLS. We confirm that in toluene there is only one fast diffusive relaxation mode in the measured intensity-intensity time correlation function even for the solution with a concentration $(C) 30$ times higher than the overlapping concentration $\left(C^{\star}\right)$. In cyclohexane, an additional slow mode appears as soon as $C / C^{\star}>1$. Our results show that the slow mode is real, not because of previously suggested artifacts in the solution preparation. The concentration, and time dependence of the characteristic relaxation time of the slow mode $\left(\langle\tau\rangle_{s}\right)$, the scaling exponent $\left(\alpha_{s}\right)$ between $\langle\tau\rangle_{s}$ and the scattering vector $(q)$, and the relative intensity contribution $\left(A_{s}\right)$ of the slow mode suggests that such a slow mode has two different natures, depending on the polymer concentration.

When $C$ is higher than the overlapping concentration $\left(C^{\star}\right)$ but lower than the entanglement concentration $\left(C_{\mathrm{e}}\right)$, only some of the chains are entangled together to form large transient clusters, which coexist with individual (nonentangled) chains in the solution. In such a transitional region between dilute and semidilute solutions, the relatively stronger segment-segment interaction in a less good/ $\theta$ solvent makes the movements of the chains inside each cluster more correlated within a short delay time window $(<1 \mathrm{~s})$ and leads to the slow mode. The temperature dependence of the slow relaxation mode in two different systems, PNIPAM/ $\mathrm{H}_{2} \mathrm{O}$ and PEO-PPO-PEO/ $\mathrm{H}_{2} \mathrm{O}$ further confirms the coexistence of individual chains and large temporal chain clusters in the semidilute regimes.

When $C>C_{\mathrm{e}}$, all the chains are entangled with each other to form one huge 'cluster'. Each chain is confined inside a 'tube' made of its surrounding chains and the gravity center of each chain segment (blob) fluctuates inside the tube within a short time scale $\left(\sim 10^{-3} \mathrm{~s}\right)$. In an athermal solvent, there is no interaction among the chains so that all the blobs are in the same microenvironment, leading to only one fast relaxation mode. In a less $\operatorname{good} / \theta$ solvent, the relatively stronger segment-segment interaction near the entanglement points makes the tube nonuniform. It can be visualized that each chain is inside a nonuniform tube with a band-like structure. Therefore, the blobs experience two different microenvironments. The restricted movement of the blobs near the entanglement points results in the slow mode. The use of a special $(2+2)$ photoaddition to induce in situ crosslinking of polymer chains rather than the entanglement in a semidilute solution further indicates an expected increase of the relative contribution of the slow mode as the crosslinking proceeds because the crosslinking enhances the restricted movement of the polymer chains near the crosslinked (entangled) points.

By using a novel $\mathrm{P}$ ('amidine'MS)-co-PS that can be in situ and reversibly switched between a neutral to a charged state by alternative bubbling of $\mathrm{CO}_{2}$ and $\mathrm{N}_{2}$, we reconfirm that when individual polymer chains are charged in salt-free dilute solutions, their translational diffusive relaxation in the neutral state splits into a fast and a slow diffusive mode $\left(D_{\mathrm{f}}\right.$ and $D_{\mathrm{s}}$ ), respectively, due to the long-range electrostatic interaction. The fast mode can be attributed to the coupled diffusion originated from a convective current generated by an induced electric field arising from fluctuation of all charged species (charges on the chain and counterions) in the solution in a short time or length scale. However, there is still no decisive evidence to differentiate whether the slow relaxation is related to large temporal aggregates formed because of the overlapping of ion clouds among different chains or to the self-diffusion of individual chains retarded by surrounding chains (interchain friction) in a long time or length 
scale. Our results indicate that the slow mode is not related to large but slowly moving scattering objects, supporting the assumption of the retarded self-diffusion. Consequently, we conclude that the slow mode observed in the semidilute, polyelectrolyte, or even gelling system is real and can be generalized as hindered motions of interacting polymer chains even though the nature of interaction can be different, including, the weak segment-segment interaction in a less good solvent to strong electrostatic interaction among polyelectrolyte chains, and even to chemical crosslinking inside gel networks.

\section{ACKNOWLEDGEMENTS}

The financial support of the National Natural Scientific Foundation of China (NNSFC) Project (20904049, 20934005 and 50773077), and the financial support of the Hong Kong Special Administration Region General Research Fund (CUHK402506, 2160291; and CUHK403908, 2160361) is gratefully acknowledged.

1 Rouse, P. E. J. A theory of the linear viscoelastic properties of dilute solutions of coiling polymers. J. Chem. Phys. 21, 1272-1280 (1953).

2 Zimm, B. H. Dynamics of polymer molecules in dilute solution-viscoelasticity, flow birefringence and dielectric loss. J. Chem. Phys. 24, 269-278 (1956).

3 Pecora, R. Spectral distribution of light scattered from flexible-coil macromolecules. J. Chem. Phys. 49, 1032-1035 (1968).

4 de Gennes, P. G. Quasi-elastic scattering of neutrons by dilute polymer solutions: I free-draining limit. Physics 3, 37-45 (1967).

5 Dubois-Violette, E. \& de Gennes, P. G. Quasi-elastic scattering by dilute, ideal, polymer solution: II effects of hydrodynamic interactions. Physics 3, 181-198 (1967).

6 Akcasu, A. Z. \& Gurol, H. Quasi-elastic scattering by dilute polymer-solutions. J. Polym. Sci.: Polym. Phys. Ed. 14, I-10 (1976).

7 Akcasu, A. Z. \& Higgins, J. S. Quasi-elastic scattering of neutrons from freely jointed polymer-chains in dilute-solutions. J. Polym. Sci.: Polym. Phys. Ed. 15, 1745-1756 (1977).

8 Benmouna, M. \& Akcasu, A. Z. Temperature effects on dynamic structure factor in dilute polymer-solutions. Macromolecules 11, 1187-1192 (1978).

9 Chu, B. Laser Light Scattering 291-293 (Academic Press, New York, 1974).

10 Berne, B. J. \& Percora, R. Dynamic Light Scattering (Wiley Interscience, New York, 1976).

11 Pecora, R. Dynamic Light Scattering: Applications of Photon Correlation Spectroscopy (Plenum Press, New York, 1985).

12 Han, C. C. \& Akcasu, A. Z. Dynamic light-scattering of dilute polymer-solutions in the non-asymptotic q-region. Macromolecules 14, 1080-1084 (1981).

13 Lodge, T. P., Han, C. C. \& Akscasu, A. Z. Temperature-dependence of dynamic light-scattering in the intermediate momentum-transfer region. Macromolecules 16, $1180-1183$ (1983)

14 King, T. A., Knox, A. \& McAdam, J. D. G. Internal motion in chain polymers. Chem. Phys. Lett. 19, 351-354 (1973).

15 Huang, W. N. \& Frederick, J. E. Determination of intramolecular motion in a randomcoil polymer by means of quasi-elastic light-scattering. Macromolecules 7, 34-39 (1974).

16 Tsunashima, Y., Nemoto, N. \& Kurata, M. Dynamic light-scattering-studies of polymersolutions .1. Histogram analysis of internal motions. Macromolecules 16, 584-589 (1983).

17 Nemoto, N., Makita, Y., Tsunashima, Y. \& Kurata, M. Dynamic light scattering studies of polymer-solutions. 3. Translational diffusion and internal motion of high molecularweight polystyrenes in benzene at infinite dilution. Macromolecules 17, 425-430 (1984).

18 Kim, S. H., Ramsay, D., Patterson, G. D. \& Selser, J. C. Static and dynamic lightscattering of poly-(alpha-methyl styrene) in toluene in the dilute region. J. Polym. Sci.Polym. Phys. 28, 2023-2056 (1990).

19 Chirico, G. \& Baldini, G. Dynamic light-scattering from DNA plasmids-diffusional and internal motion. J. Mol. Liq. 41, 327-345 (1989).

20 Chu, B., Wang, Z. \& Yu, J. Dynamic light-scattering study of internal motions of polymer coils in dilute-solution. Macromolecules 24, 6832-6838 (1991).

21 Sorlie, S. S. \& Pecora, R. A dynamic light-scattering study of a 2311 base pair DNA restriction fragment. Macromolecules 21, 1437-1449 (1988).

22 Nicolai, T., Brown, W. \& Johnsen, R. The internal-modes of polystyrene single coils studied using dynamic light-scattering. Macromolecules 22, 2795-2801 (1989).

23 Wu, C. \& Zhou, S. Internal motions of both poly( $\boldsymbol{N}$-isopropylacrylamide) linear chains and spherical microgel particles in water. Macromolecules 29, 1574-1578 (1996).

24 Tsunashima, Y. \& Kawamata, Y. Dynamics of polystyrene subchains of a styrenemethyl methacrylate diblock copolymer in solution measured by dynamic light-scattering with an isorefractive index solvent.1. dilute-solution region. Macromolecules 26, 4899-4909 (1993).
25 Jian, T., Anastasiadis, S. H., Semenov, A. N., Fytas, G., Adachi, K. \& Kotaka, T. Dynamics of composition fluctuations in diblock copolymer solutions far from and near to the ordering transition. Macromolecules 27, 4762-4773 (1994).

26 Balsara, N. P., Stepanek, P., Lodge, T. P. \& Tirrell, M. Dynamic light scattering from microstructured block copolymer solutions. Macromolecules 24, 6227-6230 (1991).

27 Pan, C., Maurer, W., Liu, Z., Lodge, T. P., Stepanek, P., von Meerwall, E. D. \& Watanabe, H. Dynamic light-scattering from dilute, semidilute, and concentrated block-copolymer solutions. Macromolecules 28, 1643-1653 (1995).

28 Chu, B. \& Nose, T. Static and dynamical properties of polystyrene in trans-decalin.4. Osmotic compressibility, characteristic lengths, and internal and pseudogel motions in the semidilute regime. Macromolecules 13, 122-132 (1980).

29 Ewen, B., Richter, D., Farago, B. \& Stühn, B. Neutron spin-echo investigations on the segmental dynamics in semidilute polymer-solutions under theta-solvent and good solvent conditions. J. Non-Cryst. Solids 172-174, 1023-1027 (1994).

30 Rital, A., Belkoura, L. \& Woermann, D. Static and dynamic light scattering experiments on semidilute solutions of polystyrene in cyclohexane between the Thetatemperature and the binodal curve. Phys. Chem. Chem. Phys. 1, 1947-1955 (1999).

31 Wiltzius, P., Hans, H. R., Cannell, D. S. \& Schaefer, D. W. Universality for static properties of polystyrenes in good and marginal solvents. Phys. Rev. Lett. 51, 1183-1186 (1983).

32 Amis, E. J. \& Han, C. C. Cooperative and self-diffusion of polymers in semi-dilute solutions by dynamic light-scattering. Polymer 23, 1403-1406 (1982).

33 Melnichenko, Y. B., Brown, W., Rangelov, S., Wignall, G. D. \& Stamm, M. Dynamic acid static correlations in solutions of polymers in liquid and supercritical solvents: dynamic light scattering and small angle neutron scattering. Phys. Lett. A 268, 186-194 (2000).

34 Adam, M. \& Delsanti, M. Dynamical properties of polymer-solutions in good solvent by Rayleigh-scattering experiments. Macromolecules 10, 1229-1237 (1977).

35 Adam, M. \& Delsanti, M. Dynamical behavior of semidilute polymer-solutions in a theta-solvent-quasi-elastic light-scattering experiments. Macromolecules 18 , 1760-1770 (1985)

36 Brown, W. \& Stepanek, P. Distribution of relaxation-times from dynamic light-scattering on semidilute solutions-polystyrene in ethyl-acetate as a function of temperature from good to theta-conditions. Macromolecules 21, 1791-1798 (1988).

37 Brown, W. \& Stepanek, P. Dynamic behavior in concentrated polystyrene cyclohexane solutions close to the theta-point-relaxation-time distributions as a function of concentration and temperature. Macromolecules 25, 4359-4363 (1992).

38 Brown, W. \& Stepanek, P. Viscoelastic relaxation in semidilute and concentrated polymer-solutions. Macromolecules 26, 6884-6890 (1993).

39 Konak, C., Mrkviekova, L. \& Bansil, R. Dynamics of pregel solutions and gels in a Theta-solvent near a spinodal. Macromolecules 29, 6158-6164 (1996).

40 Brochard, F. \& de Gennes, P. G. Dynamical scaling for polymers in theta-solvents. Macromolecules 10, 1157-1161 (1977).

41 Wang, C. H. Dynamic light-scattering, mutual diffusion, and linear viscoelasticity of polymer-solutions. J. Chem. Phys. 95, 3788-3797 (1991).

42 Wang, C. H. Dynamic light-scattering and viscoelasticity of a binary polymer-solution. Macromolecules 25, 1524-1529 (1992).

43 Wang, C. H., Fytas, G. \& Fischer, E. W. Determination of the longitudinal compliance of polyvinyl acetate) by using the dynamic light-scattering technique. J. Chem. Phys. 82, 4332-4336 (1985)

44 Wang, C. H. \& Zhang, X. Q. Quasi-elastic light-scattering and viscoelasticity of polystyrene in diethyl phthalate. Macromolecules 26, 707-714 (1993).

45 Wang, C. H. \& Zhang, X. Q. Quasi-elastic light-scattering investigation of concentration fluctuations and coupling to stress-relaxation in a polymer-solution-polystyrene in $\mathrm{CCl}_{4}$. Macromolecules 28, 2288-2296 (1995).

46 Wang, C. H., Sun, Z. \& Huang, Q. R. Quasielastic light scattering of polystyrene in diethyl malonate in semidilute concentration. J. Chem. Phys. 105, 6052-6059 (1996).

47 Adam, M., Farago, B., Schleger, P., Raspaud, E. \& Lairez, D. Binary contacts in semidilute solution: good and theta solvents. Macromolecules 31, 9213-9223 (1998).

48 Colby, R. H. \& Rubinstein, M. 2-parameter scaling for polymers in theta-solvents. Macromolecules 23, 2753-2757 (1990).

49 Nicolai, T., Brown, W., Johnsen, R. M. \& Stepanek, P. Dynamic behavior of o solutions of polystyrene investigated by dynamic light-scattering. Macromolecules 23, 1174 (1990).

50 Nicoclai, T. \& Brown, W. Static and dynamic light-scattering-studies on semidilute solutions of polystyrene in cyclohexane as a function of temperature. Macromolecules 23, 3150-3155 (1990).

51 Nicoclai, T. \& Brown, W. Cooperative diffusion of concentrated polymer solutions: a static and dynamic light scattering study of polystyrene in DOP. Macromolecules 29, 1698-1704 (1996).

52 Brown, W. \& Johnsen, R. M. Diffusion-coefficients in semidilute solutions evaluated from dynamic light-scattering and concentration gradient measurements as a function of solvent quality.1.Intermediate molecular-weights. Macromolecules 18, 379-387 (1985).

53 Xie, Y., Ludwing, K. F., Bansil, R., Gallagher, P. D., Cao, X. \& Morales, G. Small-angle $\mathrm{X}$-ray scattering studies of semidilute polystyrene-cyclohexane solutions. Physica $A$ 232, 94-108 (1996).

54 Kostko, A. F., Anisimov, M. A. \& Sengers, J. V. Dynamic crossover to tricriticality and anomalous slowdown of critical fluctuations by entanglements in polymer solutions. Phys. Rev. E 66, 020803-020806 (2002). 
55 Brown, W., Johnsen, R. M., Konak, C. \& Dvoranek, L. Dynamics in concentrated polymer-solutions by polarized Rayleigh-Brillouin scattering and dynamic light-scattering. J. Chem. Phys. 95, 8568-8577 (1991).

56 Nose, T. \& Chu, B. Static and dynamical properties of polystyrene in trans decalin. 1. NBS 705 standard near $\boldsymbol{\theta}$ conditions. Macromolecules 12, 590-599 (1979).

57 Sawatari, N., Yoshizaki, T. \& Yamakawa, H. Dynamic structure factor of polystyrene and poly(methyl methacrylate) in Theta solvents. Macromolecules 31, 4218-4222 (1998).

58 Schröder, J. M., Wiegand, S., Aberle, L. B., Kleemeier, M. \& Schroer, W. Experimental determination of singly scattered light close to the critical point in a polystyrenecyclohexane mixture. Phys. Chem. Chem. Phys. 1, 3287-3292 (1999).

59 Appelt, B. \& Meyerhoff, G. Characterization of polystyrenes of extremely high molecular-weights. Macromolecules 13, 657-662 (1980).

60 Brown, W. Slow-mode diffusion in semidilute solutions examined by dynamic lightscattering. Macromolecules 17, 66-72 (1984)

61 Brown, W. Dynamical properties of high molecular-weight polystyrene in tetrahydrofuran in the dilute semidilute transition region. Macromolecules 18, 1713-1719 (1985).

62 Bastide, J. \& Leibler, L. Large-scale heterogeneities in randomly cross-linked networks. Macromolecules 21, 2647-2649 (1988).

63 Brown, W. \& Mortensen, K. Dynamical properties of high molecular-weight polystyrene in tetrahydrofuran in the dilute semidilute transition region. Macromolecules 21, 420-425 (1988).

64 Colby, R. H., Fetters, L. J., Funk, W. G. \& Graessley, W. W. Effects of concentration and thermodynamic interaction on the viscoelastic properties of polymer-solutions. Macromolecules 24, 3873-3882 (1991).

65 Nicolai, T., Brown, W., Hvidt, S. \& Heller, K. A comparison of relaxation-time distributions obtained from dynamic light-scattering and dynamic mechanical measurements for high-molecular-weight polystyrene in entangled solutions. Macromolecules 23, 5088-5096 (1990).

66 Vshivkov, S. A. \& Safronov, A. P. The conformational coil-globule transition of polystyrene in cyclohexane solution. Macromol. Chem. Phys. 198, 3015-3023 (1997)

67 Liu, Z., Pan, C., Lodge, T. P. \& Stepanek, P. Dynamic light-scattering from blockcopolymer solutions under the zero average contrast condition. Macromolecules $\mathbf{2 8}$, 3221-3229 (1995)

68 Borsali, R., Fischer, E. W. \& Benmouna, M. Dynamic light-scattering from polystyrenepoly(methylmethacrylate) diblock copolymer in toluene. Phys. Rev. A 43, 5732-5735 (1991).

69 Benmouna, M., Benoit, H., Borsali, R. \& Duval, M. Theory of dynamic scattering from copolymer solutions using the random phase approximation. Macromolecules 20, 2620-2624 (1987)

70 Jian, T., Anastasiadis, S. H., Semenov, A. N., Fytas, G., Adachi, K. \& Kotaka, T. Dynamics of composition fluctuations in diblock copolymer solutions far from and near to the ordering transition. Macromolecules 27, 4762-4773 (1994).

71 Semenov, A. N., Anastasiadis, S. H., Boudenne, N., Fytas, G., Xenidou, M. \& Hadjichristidis, N. Dynamic structure factor of diblock copolymers in the ordering regime. Macromolecules 30, 6280-6294 (1997).

72 Chrissopoulou, K., Pryamitsyn, V. A., Anastasiadis, S. H., Fytas, G., Semenov, A. N., Xenidou, M. \& Hadjichristidis, N. Dynamics of the most probable composition fluctuations of 'real' diblock copolymers near the ordering transition. Macromolecules 34, 2156-2171 (2001)

73 Anastasiadis, S. H., Fytas, G., Vogt, S. \& Fisher, E. W. Breathing and composition pattern relaxation in homogeneous diblock copolymers. Phys. Rev. Lett. 70, 2415-2418 (1993).

74 Boudenne, N., Anastasiadis, S. H., Fytas, G., Xenidou, M., Hadjichristidis, N., Semenov, A. N. \& Fleischer, G. Thermodynamic effects on internal relaxation in diblock copolymers. Phys. Rev. Lett. 77, 506-509 (1996).

$75 \mathrm{Li}$, J. F., Li, W., Huo, H., Luo, S. Z. \& Wu, C. Reexamination of the slow mode in semidilute polymer solutions: the effect of solvent quality. Macromolecular 41, 901-911 (2008).

76 Sedlak, M., Konak, C., Stepanek, P. \& Jakes, J. Semidilute solutions of poly (methacrylic acid) in the absence of salt-dynamic light-scattering study. Polymer 28, 873-880 (1987).

77 Schmitz, K. S. \& Yu, J. W. On the electrostatic contribution to the persistence length of flexible poly-electrolytes. Macromolecules 21, 484-493 (1988).

78 Mattoussi, M., Karasz, F. E. \& Langley, K. H. Electrostatic and screening effects on the dynamic aspects of polyelectrolyte solutions. J. Chem. Phys. 93, 3593-3603 (1990)

79 Forster, S., Schmidt, M. \& Antonietti, M. Static and dynamic light-scattering by aqueous polyelectrolyte solutions - effect of molecular-weight, charge-density and added salt. Polymer 31, 781-792 (1990).

80 Sedlak, M. \& Amis, E. J. Dynamics of moderately concentrated salt-free polyelectrolyte solutions-molecular-weight dependence. J. Chem. Phys. 96, 817-825 (1992).

81 Ermi, B. D. \& Amis, E. J. Model solutions for studies of salt-free polyelectrolytes. Macromolecules 29, 2701-2703 (1996).

82 Topp, A., Belkoura, L. \& Woermann, D. Effect of charge density on the dynamic behavior of polyelectrolytes in aqueous solution. Macromolecules 29, 5392-5397 (1996).

83 Nierling, W. \& Nordmeeir, E. Studies on polyelectrolyte solutions. 7. Fast, heterogeneous, and slow diffusion modes of poly(diallyl- $\boldsymbol{N}, \boldsymbol{N}$-dimethylammonium chloride) in aqueous alcoholic salt solvents. Pol. J. 29, 795-806 (1997).
84 Valachovic, D. E. \& Amis, E. J. Polyelectrolyte dendrimers in low-salt solutions. Abstr. Pap. Am. Chem. Soc. 209, 58 (1995).

85 Dobrynin, A. V. \& Rubinstein, M. Theory of polyelectrolytes in solutions and at surfaces. Prog. Polym. Sci. 30, 1049-1118 (2005).

86 Muthukumar, M. Polyelectrolyte dynamics. Adv. Chem. Phys. 131, 1-60 (2005)

87 Muthukumar, M. Double screening in polyelectrolyte solutions: limiting laws and crossover formulas. J. Chem. Phys. 105, 5183-5199 (1996).

88 Muthukumar, M. Dynamics of polyelectrolyte solutions. J. Chem. Phys. 107, 2619-2635 (1997)

$89 \mathrm{Li}, \mathrm{X}$. \& Reed, W. F. Polyelectrolyte properties of proteoglycan monomers. J. Chem. Phys. 94, 4568-4580 (1991)

90 Reed, W. F., Ghosh, S., Medjahdi, G. \& Francois, J. Dependence of polyelectrolyte apparent persistence lengths, viscosity, and diffusion on ionic-strength and linear charge-density. Macromolecules 24, 6189-6198 (1991).

91 Ghosh, S., Li, X., Reed, C. E. \& Reed, W. F. Apparent persistence lengths and diffusion behavior of high-molecular-weight hyaluronate. Biopolymers 30, 1101-1112 (1990).

92 Peitzsch, R. M., Burt, M. J. \& Reed, W. F. Evidence of partial draining for linear polyelectrolytes-heparin, chondroitin 6-sulfate, and poly(styrenesulfonate). Macromolecules 25, 806-815 (1992).

93 Ghosh, S., Peitzsch, R. M. \& Reed, W. F. Aggregates and other particles as the origin of the extraordinary diffusional phase in polyelectrolyte solutions. Biopolymers $\mathbf{3 2}$, 1105-1122 (1992)

94 Ermi, B. D. \& Amis, E. J. Domain structures in low ionic strength polyelectrolyte solutions. Macromolecules 31, 7378-7384 (1998).

95 Cong, R., Temyanko, E., Russo, P. S., Edwin, N. \& Uppu, R. M. Dynamics of poly (styrenesulfonate) sodium salt in aqueous solution. Macromolecules 39, 731-739 (2006).

96 Yuan, G. C., Wang, X. H., Han, C. C. \& Wu, C. Reexamination of slow dynamics in semidilute solutions: from correlated concentration fluctuation to collective diffusion. Macromolecules 39, 3642-3647 (2006).

97 Yuan, G. C., Wang, X. H., Han, C. C. \& Wu, C. Reexamination of slow dynamics in semidilute solutions: temperature and salt effects on semidilute poly( $N$-isopropylacrylamide) aqueous solutions. Macromolecules 39, 6207-6209 (2006).

98 Li, W., Hong, L. Z., Ngai, T., Huang, H. Y., He, T. B. \& Wu, C. A comparative study of chain dynamics of di- and tri-block copolymers in semidilute solution in a nonselective solvent. Chin. J. Polym. Sci. 22, 589-598 (2004).

$99 \mathrm{Li}$, J. F., Lu, Y. J., Zhang, G. Z., Li, W. \& Wu, C. A slow relaxation mode of polymer chains in a semidilute solution. Chin. J. Polym. Sci. 26, 465-473 (2008).

$100 \mathrm{Wu}$, C. \& Ngai, T. Reexamination of slow relaxation of polymer chains in sol-gel transition. Polymer 45, 1739-1742 (2004).

101 Ngai, T., Wu, C. \& Chen, Y. Origins of the speckles and slow dynamics of polymer gels. J. Phys. Chem. B. 108, 5532-5540 (2004).

102 Ngai, T., Wu, C. \& Chen, Y. Effects of temperature and swelling on chain dynamics during the sol-gel transition. Macromolecules 37, 987-993 (2004).

103 Ngai, T. \& Wu, C. Effect of cross-linking on dynamics of semidilute copolymer solutions: poly(methyl methacrylate-co-7-acryloyloxy-4-methylcoumarin) in chloroform. Macromolecules 36, 848-854 (2003).

104 Zhao, Y. \& Wu, C. A hybrid polymer gel and its static nonergodicity. Chin. J. Polym. Sci. 20, 269-274 (2002).

105 Zhao, Y., Zhang, G. Z. \& Wu, C. Nonergodic dynamics of a novel thermally sensitive hybrid gel. Macromolecules 34, 7804-7808 (2001).

106 Wang, C. Q., Jiang, S. H. \& Wu, C. Application of the temperature-ramped holographic relaxation spectroscopy in the investigation of physically cross-linked gels. Macromolecules 34, 6737-6741 (2001).

107 Zhou, K. J., Li, J. F., Lu, Y. J., Zhang, G. Z. \& Wu, C. Re-examination of dynamics of polyeletrolytes in salt-free dilute solutions by designing and using a novel neutralcharged-neutral reversible polymer. Macromolecules 42, 7146-7154 (2009).

108 Chu, B. Laser Light Scattering 2nd edn. 84-85 (Academic Press, New York, 1991).

109 Huglin, M. B. Light Scattering from Polymer Solution (Academic Press, New York, 1972)

110 Kuznetsova, N. A. \& Kaliya, O. L. Photochemistry of coumarins. Uspekhi Khimii 61, 1243-1267 (1992).

111 Lin, S. C., Lee, W. I. \& Schurr, J. M. Brownian-motion of highly charged poly (I-lysine)—effects of salt and polyion concentration. Biopolymers 17, 1041-1064 (1978)

112 Brown, W. \& Nicolai, T. Static and dynamic behavior of semidilute polymer-solutions. Colloid Polym. Sci. 268, 977-990 (1990).

113 Teraoka, I. Polymer Solution (John Wiley Sons: Inc., New York, 2002).

114 de Gennes, P. G. Dynamics of entangled polymer solutions. I. The Rouse Model. Macromolecules 9, 587-593 (1976).

115 de Gennes, P. G. Dynamics of entangled polymer solutions. II. Inclusion of hydrodynamic interactions. Macromolecules 9, 594-598 (1976).

116 Daoud, M., Cotton, J. P., Farnoux, B., Jannink, G., Sarma, G., Benoit, H., Duplessix, R., Picot, C. \& de Gennes, P. G. Solutions of flexible polymers-neutron experiments and interpretation. Macromolecules 8, 804-818 (1975).

117 de Gennes, P. G. Scalling Concenpts in Polymer Physicas (Cornell University Press, Ithaca, NY, 1979).

118 Koike, A., Nemoto, N., Inoue, T. \& Osaki, K. Dynamic light-scattering and dynamic viscoelasticity of poly(vinyl alcohol) in aqueous borax solutions. 1. Concentrationeffect. Macromolecules 28, 2339-2344 (1995).

119 Tao, H., Huang, C. \& Lodge, T. P. Correlation length and entanglement spacing in concentrated hydrogenated polybutadiene solutions. Macromolecules 32, 1212-1217 (1999) 
120 Yamamoto, I., Iwasaki, K. \& Hirotsu, S. Light-scattering study of condensation of poly (N-isopropylacrylamide) chain. J. Phys. Soc. Jpn. 51, 210-215 (1989).

121 Di Marzio, E. A. The ten classes of polymeric phase transitions: their use as models for self-assembly. Prog. in Polym. Sci. 24, 329-377 (1999) and the references in it.

122 Smart, T., Lomas, H., Massignani, M., Flores-Merino, M. V., Perez, L. R. \& Battaglia, G. Block copolymer nanostructures. Nano Today. 3, 38-46 (2008) and the references in it.

123 Tanaka, T., Hocker, L. O. \& Benedek, G. B. Spectrum of light scattered from a viscoelastic gel. J. Chem. Phys. 59, 5151-5159 (1973).

124 Panyukov, S. \& Rabin, Y. Polymer gels: frozen inhomogeneities and density fluctuations. Macromolecules 29, 7690-7975 (1996).

125 Pusey, P. N. Dynamic light-scattering by nonergodic media. Macromol. Symp. 79, 17-30 (1994).

126 Wu, C., Zuo, J. \& Chu, B. Molecular-weight distribution of a branched epoxy polymer1,4-butanediol diglycidyl ether with cis-1,2-cyclohexanedicarboxylic anhydride. Macromolecules 22, 633-639 (1989).

$127 \mathrm{Wu}, \mathrm{C} ., \mathrm{Ju}, \mathrm{Z}$. \& Chu, B. Laser-light scattering studies of epoxy polymerization of 1,4butanediol diglycidyl ether with cis-1,2-cyclohexanedicarboxylic anhydride. Macromolecules 22, 838-842 (1989).

128 Shibayama, M. Spatial inhomogeneity and dynamic fluctuations of polymer gels. Macromol. Chem. Phys. 199, 1-30 (1998) and the references in it.

129 Panyukov, S. \& Rabin, Y. Statistical physics of polymer gels. Phys. Rep. 269, 1-131 (1996).

130 Onuki, A. Theory of phase-transition in polymer gels. Adv. Polym. Sci. 109, 63-121 (1993).

131 Konak, C., Helmstedt, M. \& Bansil, R. Dynamics in solutions of associating statistical copolymers. Macromolecules 30, 4342-4346 (1997).

132 Bansil, R., Nie, H. F., Konak, C., Helmstedt, M. \& Lal, J. Structure and dynamics of solutions of a polystyrene-polybutadiene pentablock copolymer in a styrene-selective solvent. J. Poly. Sci.B Polym. Phys. 40, 2807-2816 (2002).

133 Zheng, H. \& Teraoka, I. Dynamic light scattering from semidilute solutions of a styrene-acrylonitrile random copolymer. Macromolecules 34, 6074-6082 (2001).

134 Vogt, S., Anastasiadis, S. H., Fytas, G. \& Fischer, E. W. Dynamics of composition fluctuations in diblock copolymer melts above the ordering transition. Macromolecules 27, 4335-4343 (1994)
135 Chrissopoulou, K., Pryamitsyn, V. A., Anastasiadis, S. H., Fytas, G., Semenov, A. N., Xenidou, M. \& Hadjichristidis, N. Dynamics of the most probable composition fluctuations of 'real' diblock copolymers near the ordering transition. Macromolecules 34, 2156-2171 (2001)

136 Jian, T., Anastasiadis, S. H., Semenov, A. N., Fytas, G., Adachi, K. \& Kotaka, T. Dynamics of composition fluctuations in diblock copolymer solutions far from and near to the ordering transition. Macromolecules 27, 4762-4773 (1994).

137 Konak, C., Helmstedt, M. \& Bansil, R. Temperature dependence of dynamics of solutions of triblock copolymer in a selective solvent. Polymer 41, 9311-9315 (2000).

138 Nystrom, B. \& Kjoniksen, A. L. Dynamic light scattering of a poly(ethylene oxide) poly(propylene oxide) poly(ethylene oxide) triblock copolymer in water. Langmuir 13, 4520-4526 (1997).

139 Konak, C., Fleischer, G., Tuzar, Z. \& Bansil, R. Dynamics of solutions of triblock copolymers in a selective solvent: effect of varying copolymer concentration. J. Poly. Sci.B Polym. Phys. 38, 1312-1322 (2000).

140 Raspaud, E., Lairez, D., Adam, M. \& Carton, J. P. Triblock copolymers in a selective solvent. 2. Semidilute solutions. Macromolecules 29, 1269-1277 (1996).

141 Konak, C., Helmstedt, M. \& Bansil, R. Temperature dependence of dynamics in solutions of associating random copolymers. Macromolecules 31, 4639-4641 (1998).

142 Alexandridis, P., Holzwarth, J. F. \& Hatton, T. A. Micellization of poly(ethylene oxide)poly(propylene oxide)-poly(ethylene oxide) triblock copolymers in aqueous solutionsthermodynamics of copolymer association. Macromolecules 27, 2414-2425 (1994).

143 Moussaid, A., Munch, J. P., Schosseler, F. \& Candau, S. J. Light scattering study of partially ionized poly(acrylic acid) systems : comparison between gels and solutions. J. Phys. II. 1, 637-650 (1991).

144 Krall, A. H., Huang, Z. \& Weitz, D. A. Dynamics of density fluctuations in colloidal gels. Physica A 235, 19-33 (1997).

145 Norisuye, T., Inoue, M., Shibayama, M., Tamaki, R. \& Chujo, Y. Time-resolved dynamic light scattering study on the dynamics of silica gels during gelation process. Macromolecules 33, 900-905 (2000).

146 Shibayama, M. \& Norisuye, T. Gel formation analyses by dynamic light scattering. Bull. Chem. Soc. Jpn. 75, 641-659 (2002).

147 Pusey, P. N. \& Tough, R. J. A. Dynamic light-scattering, a probe of Brownian particle dynamics. Adv. Colloid Interface Sci. 16, 143-159 (1982).

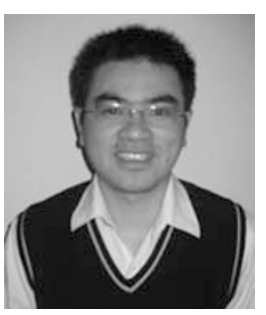

Junfang Li was born in Hubei province of China in 1979. He received a BS in physical chemistry from the Department of Chemical Physics at the University of Science and Technology of China (USTC) in 2002, and a PhD in the same university in 2007. His PhD thesis work, under the direction of Professor $\mathrm{Chi} \mathrm{Wu}$, was about the using a combination of synthetic chemistry and laser light scattering to study the dynamics and structures of polymer solutions and gel networks. After a 2-year postdoctoral fellowship in the Department of Mechanics at USTC (2007-2009), he joined the Shanghai Institute of Organic Chemistry (SIOC), Chinese Academy of Sciences(CAS), as a research assistant in the State Key Laboratory of Organometallic Chemistry. His current research interests include polyelectrolyte, coordination polymerization, immobilizing of polyolefin catalyzer and characterizing of polyolefin synthesized by new type single-site catalyst.

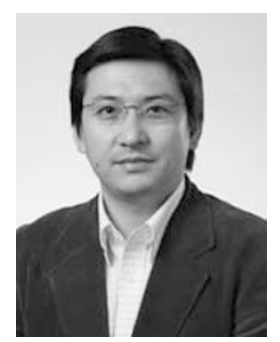

To Ngai was born in Fujian province of China in 1976. He received his BS in chemistry at the Chinese University of Hong Kong in 1999. In 2003, he obtained his $\mathrm{PhD}$ in chemistry in the same university under the supervision of Professor Chi Wu. He moved to BASF (Ludwigshafen, Germany) in 2003; first as a postdoctoral fellow for 1 year, then as the chemist in polymer physics division. In July 2005, he went to Professor Lodge's group in the Chemistry Department of the University of Minnesota, working on polymer blending. He returned Hong Kong in 2006 and joined the Chemistry Department of the Chinese University of Hong Kong as a research assistant professor and is now assistant professor. Currently, his research interests center on the designing, synthesizing and measuring interactions between polymers, particles and soft materials.

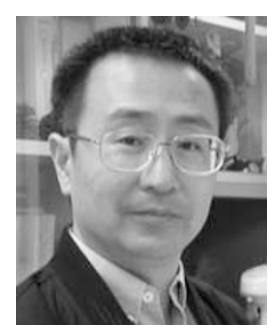

Chi Wu was born in Wuhu, Anhui, China (1955). He graduated from the Department of Chemical Physics at the University of Science and Technology of China in 1982. It was there that he received a rigorous training in physics, but in a chemistry department. After obtaining his $\mathrm{PhD}$ in 1987 followed by a 2-year postdoctoral experience, both under the supervision of Professor Benjamin Chu in the State University of New York at Stony Brook, he moved to BASF (Ludwigshafen, Germany) in 1989; first as an Alexander von Humboldt Fellow for 1 year, then as the supervisor of BASF's laser light-scattering laboratory. He joined the Chinese University of Hong Kong in 1992 and is now Professor of Chemistry. His research combines synthetic chemistry, polymer physics and molecular biology to design and execute decisive experiments to answer important questions in macromolecules, colloids and biology. Among these, include the development of nonviral vectors for molecular medicines; the nucleation of neuron-degenerative disease-related protein aggregation; the design, synthesis and assembly of functional macromolecules; the dynamics and structures of polymer solutions and gel networks; and molecular characteristic properties of intractable and special polymers. 